\title{
The Carbene Reactivity Surface: A Classification
}

\author{
Jean-Luc Mieusset and Udo H. Brinker \\ Institut für Organische Chemie, Universität Wien, Währinger Straße 38, A-1090 Wien, \\ Austria \\ jeanluc.mieusset@univie.ac.at; udo.brinker@univie.ac.at
}

Corresponding author. Phone: +43-1-4277-52121. Fax: +43-1-4277-52140

\section{Supplementary Information}

Cartesian coordinates and thermodynamic data for all relevant stationary points (B3LYP/6-

$31 \mathrm{G}(\mathrm{d})$ geometries):

Complete reference 11: 
Acetonitrile, NImag $=0, \mathrm{C} 3 \mathrm{~V}$

$\mathrm{C}, 0,0.0 ., 1.1807056237$

$\mathrm{H}, 0,1.0264134324,-0.0000000082,1.5610660301$

$\mathrm{H}, 0,-0.5132067233,-0.8889001032,1.5610660301$

$\mathrm{H}, 0,-0.5132067092,0.8889001114,1.5610660301$

$\mathrm{C}, 0,0 ., 0 .,-0.2804273018$

$\mathrm{N}, 0,0.0 \ldots,-1.4406954317$

Sum of electronic and zero-point Energies=

Sum of electronic and thermal Energies=

Sum of electronic and thermal Enthalpies=

Sum of electronic and thermal Free Energies=

$-132.709286$

$-132.705692$

$-132.704748$

$-132.732265$

$-40.473175$

$-40.470311$

$-40.469367$

$-40.490499$

Isobutane, NImag $=0, \mathrm{C} 3 \mathrm{~V}$

$\mathrm{C}, 0,0 ., 0 .,-0.3723765994$

$\mathrm{H}, 0,0 ., 0 .,-1.4729790046$

C, $0,1.2663648077,-0.7311360633,0.0957854298$

$\mathrm{H}, 0,1.3176204616,-0.7607285289,1.192305029$

$\mathrm{H}, 0,1.286987272,-1.7663800103,-0.2656357037$

$\mathrm{H}, 0,2.1732235975,-0.2313736685,-0.2656357037$

C, $0,0.0000000006,1.4622721256,0.0957854298$

$\mathrm{H}, 0,0.8862363257,1.9977536771,-0.2656357037$

$\mathrm{H}, 0,-0.886236324,1.9977536778,-0.2656357037$

$\mathrm{H}, 0,0.0000000006,1.5214570568,1.192305029$

$\mathrm{C}, 0,-1.2663648083,-0.7311360623,0.0957854298$

$\mathrm{H}, \mathrm{0},-2.1732235977,-0.2313736668,-0.2656357037$

$\mathrm{H}, \mathrm{O},-1.2869872735,-1.7663800093,-0.2656357037$

$\mathrm{H}, 0,-1.3176204622,-0.7607285278,1.192305029$

Sum of electronic and zero-point Energies=

Sum of electronic and thermal Energies=

Sum of electronic and thermal Enthalpies=

Sum of electronic and thermal Free Energies=

$-158.326419$

$-158.320742$

$-158.319797$

$-158.352992$

Dibromocarbene (1), NImag $=0$

$\mathrm{C}, 0,0,0 ., 1.0213482629$

$\mathrm{Br}, 0,0.1 .5661782351,-0.0875441368$

$\mathrm{Br}, 0,0 .,-1.5661782351,-0.0875441368$

Sum of electronic and zero-point Energies=

Sum of electronic and thermal Energies=

Sum of electronic and thermal Enthalpies=

5181.397346

$-5181.393626$

$-5181.392682$

Sum of electronic and thermal Free Energies=

$-5181.425500$

Insertion of 1 into acetonitrile, NImag=1, $-860.3 \mathrm{~cm}^{-1}$

C, $0,-1.6714157065,-1.5117452438,0.7861872878$

C, $0,0.4483738212,-0.0902876017,0.8381653004$ 
$\mathrm{H}, 0,-0.4242471514,-0.7630780304,1.2969163575$ $\mathrm{H}, 0,-2.0770753807,-1.7102824965,1.7789855408$ C, $0,-2.4461713549,-0.6676563137,-0.0349705587$ $\mathrm{N}, 0,-2.9957360955,0.1148146387,-0.7092154247$ $\mathrm{H}, 0,-1.2177876488,-2.3721791685,0.2983517706$ $\mathrm{Br}, 0,-0.1873292947,1.5529035061,0.1913252586$ $\mathrm{Br}, 0,1.5217447888,-1.0483328724,-0.4183549121$

Sum of electronic and zero-point Energies= Sum of electronic and thermal Energies= Sum of electronic and thermal Enthalpies= Sum of electronic and thermal Free Energies=

$-5314.085107$

$-5314.077562$

$-5314.076618$

$-5314.120220$

Insertion of 1 into methane, NImag=1, $-1142.5 \mathrm{~cm}^{-1}$ C, $0,-0.0000381745,2.3458666612,-0.0305815739$ $\mathrm{C}, 0,0.0000254626,0.2636550265,0.7999050021$ $\mathrm{H}, 0,0.0000121406,1.4089759244,1.0551489745$ $\mathrm{H}, 0,-0.9199479891,2.8627503357,0.2451375188$ $\mathrm{H}, 0,0.9198740544,2.8627783859,0.2450763009$ $\mathrm{H}, 0,-0.0000667371,1.9719474467,-1.0481183298$ $\mathrm{Br}, 0,-1.6045099031,-0.3537949104,-0.0729834286$ $\mathrm{Br}, 0,1.6045157546,-0.3537360102,-0.0731075724$

Sum of electronic and zero-point Energies= Sum of electronic and thermal Energies= Sum of electronic and thermal Enthalpies= Sum of electronic and thermal Free Energies=

$-5221.837911$

$-5221.831948$

$-5221.831003$

$-5221.869850$

Insertion of 1 into isobutane, NImag=1, $-727.7 \mathrm{~cm}^{-1}$ C, $0,-1.9551193286,0.0002612857,-0.026103176$

$\mathrm{C}, 0,0.4275378459,0.0002183445,-0.8437313835$

$\mathrm{H}, 0,-0.6696450058,0.0004414998,-1.0818282744$

C, $0,-2.4938126226,1.2880211717,-0.5925639357$

$\mathrm{H}, 0,-1.8360402267,2.1305551032,-0.3513590277$

$\mathrm{H}, 0,-3.4792894878,1.5060388499,-0.1501260328$

$\mathrm{H}, 0,-2.6248473508,1.2400816365,-1.6792033022$

C, $0,-2.4941342982,-1.286995355,-0.5934031003$

$\mathrm{H}, 0,-3.4796578032,-1.5050625622,-0.1510943709$

$\mathrm{H}, 0,-1.8365634144,-2.1298475045,-0.3527605558$

$\mathrm{H}, \mathrm{O},-2.6251706369,-1.2383104282,-1.6800089751$

C, $0,-1.6601525627,-0.0002570312,1.4410350848$

$\mathrm{H}, 0,-1.097120938,-0.8908938287,1.7394218486$

$\mathrm{H}, 0,-2.6074722686,-0.0003459316,2.0047754969$

$\mathrm{H}, 0,-1.0969327868,0.890060992,1.7400168913$

$\mathrm{Br}, 0,1.0060283509,1.6033244505,0.0526646236$

$\mathrm{Br}, 0,1.005595241,-1.6036161168,0.0516429583$

Sum of electronic and zero-point Energies=

Sum of electronic and thermal Energies=

Sum of electronic and thermal Enthalpies=

Sum of electronic and thermal Free Energies=

$-5339.713663$

$-5339.703450$

$-5339.702506$

$-5339.750499$

Acetylcarbene (2), NImag $=0$

C, $0,0.4001223333,-0.3756738355,1.3095703864$

$\mathrm{H}, 0,0.354799294,0.3545724306,2.1191965448$

C, $0,-0.0192971081,-0.0157306232,0.0547281001$

$0,0,1.0906915705,0.5368979187,-0.2261797925$

$\mathrm{C}, 0,-1.1680039104,-0.2418540345,-0.8638208071$

$\mathrm{H}, 0,-0.8115399567,-0.6737463731,-1.8052376538$

$\mathrm{H}, \mathrm{O},-1.6324998387,0.723781413,-1.0966082138$ 
$\mathrm{H}, 0,-1.9132199514,-0.9002398615,-0.4107784132$

Sum of electronic and zero-point Energies=

$-191.747532$

Sum of electronic and thermal Energies=

$-191.742439$

Sum of electronic and thermal Enthalpies=

$-191.741494$

Sum of electronic and thermal Free Energies=

$-191.775458$

Insertion of 2 into acetonitrile, NImag=1, $-223.3 \mathrm{~cm}^{-1}$ C, $0,1.434977827,0.1306523357,-0.4760638094$ $\mathrm{C}, 0,-0.8566073626,0.9516221921,0.1447374707$

$\mathrm{H}, 0,-0.8846887077,1.7261057945,-0.6308560488$

$\mathrm{H}, 0,0.6563590725,0.6481770796,0.2083483366$

$\mathrm{H}, 0,1.0653337792,-0.8814064795,-0.6568595929$

C, $0,-1.5483390961,-0.2428688505,-0.1508973315$

C, $0,-3.0409721212,0.1875561326,0.0452537694$

$\mathrm{H}, \mathrm{O},-3.4267769306,-0.3793279252,0.8963174576$

$\mathrm{H}, 0,-3.5686258747,-0.1031708995,-0.8684689234$

$\mathrm{H}, \mathrm{0},-3.2032561422,1.2539634659,0.2356193537$

$0,0,-1.2323903992,-1.4043012994,-0.3647195883$

$\mathrm{C}, 0,2.6779739785,0.1515430493,0.2735252438$

$\mathrm{N}, 0,3.6712177577,0.1730102239,0.8750838845$

$\mathrm{H}, 0,1.5200543399,0.6879686374,-1.4112631263$

Sum of electronic and zero-point Energies=

Sum of electronic and thermal Energies=

Sum of electronic and thermal Enthalpies=

Sum of electronic and thermal Free Energies=

$-324.452860$

$-324.444302$

$-324.443358$

$-324.487062$

Insertion of 2 into acetonitrile, NImag=1, $-291.8 \mathrm{~cm}^{-1}$ C, $0,1.2156734071,-0.9273805025,0.7881533308$

$\mathrm{H}, 0,0.4948636757,-1.5511539755,0.2532592505$

$\mathrm{H}, 0,1.4856975878,-1.3994702302,1.7386660704$

C, $0,-0.3755547951,0.9934537064,0.4573478588$

$\mathrm{H}, 0,0.2721408734,1.4848733629,-0.2782919534$

$\mathrm{H}, 0,0.6788910647,0.0306974365,1.1325556401$

$\mathrm{C}, 0,2.3865657425,-0.6025589537,-0.0057481348$

$\mathrm{N}, 0,3.3151504987,-0.3282576515,-0.6469166544$

C, $0,-1.4480001658,0.2405239482,-0.0572306087$

$0,0,-1.7454355481,-0.9440574741,-0.1096831632$

C, $0,-2.4528276159,1.370894963,-0.469483383$

$\mathrm{H}, 0,-3.3312164006,1.2662209477,0.1722258352$

$\mathrm{H}, 0,-2.0759520842,2.3953989742,-0.3789963899$

$\mathrm{H}, 0,-2.7221332597,1.1740978693,-1.5117709443$

Sum of electronic and zero-point Energies=

Sum of electronic and thermal Energies=

Sum of electronic and thermal Enthalpies=

Sum of electronic and thermal Free Energies=

$-324.454646$

$-324.446095$

$-324.445151$

$-324.488584$

Insertion of 2 into methane, NImag=1, $-280.2 \mathrm{~cm}^{-1}$ C, $0,0.2687704637,-1.0095478278,-0.4133874552$

$\mathrm{C}, 0,-0.7774081755,-0.1163989554,-0.0224965964$

$\mathrm{H}, 0,1.5573563939,-0.3646404656,-0.5894432467$

C, $0,2.3570712354,0.0898822201,0.139706059$

$\mathrm{H}, 0,0.5054595499,-1.801752045,0.2999088574$

$0,0,-1.843193442,-0.741035351,0.0935646125$

$\mathrm{C}, 0,-0.6963051416,1.3912164993,0.0348069172$

$\mathrm{H}, 0,-0.1064781735,1.7892525334,-0.7974718051$

$\mathrm{H}, 0,-0.2180797575,1.714662716,0.9678156751$

$\mathrm{H}, 0,-1.7089280198,1.8004905104,-0.0010167497$ 
$\mathrm{H}, 0,3.2157787599,-0.5768517786,0.0413241497$ $\mathrm{H}, 0,2.5593673592,1.0791954393,-0.2757135847$ $\mathrm{H}, 0,2.028301132,0.1570142809,1.1743062567$

Sum of electronic and zero-point Energies= Sum of electronic and thermal Energies= Sum of electronic and thermal Enthalpies= Sum of electronic and thermal Free Energies=

$-232.215510$

$-232.208353$

$-232.207409$

$-232.246143$

Insertion of 2 into isobutane, frozen atoms C, $0,-0.7579189782,-1.0201208872,-0.364767832$ C, $-1,-1.7156900321,-0.3597808386,0.473805557$ $\mathrm{H},-1,0.3840588049,-0.2271406596,-0.590894871$ C, $-1,1.3937379049,0.3198169694,-0.431806491$ C, $0,1.6696662848,0.4654137534,1.0590188012$ $\mathrm{H}, 0,1.8429901034,-0.5054268804,1.5359985204$ $\mathrm{H}, 0,0.8317349045,0.9606585601,1.5575930906$ $\mathrm{H}, 0,2.5719441564,1.0748467031,1.205480983$ C, $0,2.3912511399,-0.5630478717,-1.1706471733$ $\mathrm{H}, 0,2.5053741498,-1.538527798,-0.6856047561$ $\mathrm{H}, 0,3.3749567557,-0.0747472378,-1.1737485396$ $\mathrm{H}, \mathrm{O}, 2.0897007963,-0.724339538,-2.2105925851$ $\mathrm{C}, 0,1.1125465286,1.653652448,-1.1191470914$ $\mathrm{H}, 0,0.8423485367,1.5107320868,-2.1704197979$ $\mathrm{H}, 0,2.0119628469,2.2830150927,-1.0796455805$ $\mathrm{H}, 0,0.295053627,2.1754951163,-0.6145823411$ $\mathrm{H}, 0,-0.2677830646,-1.8994603874,0.0701809658$ $\mathrm{C}, 0,-2.9146818808,-1.3097761842,0.686553252$ $\mathrm{H}, \mathrm{O},-2.7873178865,-2.3009213239,0.2384684426$ $\mathrm{H}, \mathrm{0},-3.801871655,-0.8394585882,0.2521684179$ $\mathrm{H}, 0,-3.0675373528,-1.4162882024,1.7660096773$ $0,0,-1.7373850656,0.8005773403,0.8876917798$

Sum of electronic and zero-point Energies= Sum of electronic and thermal Energies= Sum of electronic and thermal Enthalpies= Sum of electronic and thermal Free Energies=

$-350.081847$ $-350.070988$ $-350.070044$ $-350.118091$

Insertion of 2 into isobutane, frozen atoms C, $0,-0.6918253578,-1.1737548036,-0.1102576908$ C, $-1,-1.78182337,-0.4832695272,0.5002630486$ $\mathrm{H},-1,0.305450871,-0.3420506752,-0.5666818844$ C, $-1,1.317582043,0.2011552468,-0.4102891884$ C, $0,1.4310053137,0.8732749339,0.9525565467$ $\mathrm{H}, 0,1.3840403852,0.1466597777,1.7693781374$ $\mathrm{H}, 0,0.6467227017,1.6203190094,1.1073256047$ $\mathrm{H}, 0,2.3975521156,1.3916667642,1.0202192893$ C, $0,2.3543234636,-0.8998578988,-0.6181874261$ $\mathrm{H}, 0,2.3457933948,-1.6225404836,0.2045034803$ $\mathrm{H}, 0,3.3579746973,-0.4577235781,-0.6643232425$ $\mathrm{H}, 0,2.1770710601,-1.441026235,-1.5530786426$ C, $0,1.286251809,1.2002182688,-1.5685991421$ $\mathrm{H}, 0,1.1258034006,0.6951442328,-2.5264722625$ $\mathrm{H}, 0,2.2469314448,1.7298320563,-1.6194160955$ $\mathrm{H}, 0,0.5001704182,1.9507912001,-1.4364741458$ $\mathrm{H}, \mathrm{0},-0.1247775943,-1.8417438271,0.5401165393$ $0,0,-2.5772519557,-1.2637264706,1.0509492122$ C, $0,-2.106212014,0.9854584858,0.324450652$ $\mathrm{H}, \mathrm{O},-1.5914705753,1.4315048314,-0.5312206224$ $\mathrm{H}, \mathrm{0},-1.8215069688,1.5356134792,1.2300162163$ $\mathrm{H}, 0,-3.1875510307,1.0940169781,0.1988931312$ 
Sum of electronic and zero-point Energies= Sum of electronic and thermal Energies=

Insertion of 2 into isobutane, NImag=1, $-450.1 \mathrm{~cm}^{-1}, \mathrm{HF} / 3-21 \mathrm{G} *$ C, $0,-1.3353377181,0.162657985,0.0024751027$ $\mathrm{C}, 0,0.7917406991,0.8189501669,-0.6377597841$ $\mathrm{H}, 0,0.7389213889,1.7904351874,-0.1827742853$ $\mathrm{H}, 0,-0.3933730757,0.404986256,-0.7183289851$ C, $0,1.6416931624,-0.202166835,-0.1405649906$ $0,0,1.3389177139,-1.355756249,0.168191$ C, $0,3.1013781955,0.2586211167,-0.1091631379$ $\mathrm{H}, 0,3.6644526048,-0.3481132727,-0.8074331467$ $\mathrm{H}, 0,3.4949405314,0.0814420587,0.8845787205$ $\mathrm{H}, 0,3.2220440716,1.3004180689,-0.3720551773$ C, $0,-1.7358796791,-1.1856242647,-0.6033606621$ $\mathrm{H}, 0,-2.6531341847,-1.5221610018,-0.1288065382$ $\mathrm{H}, 0,-0.9473063354,-1.9004594588,-0.426307501$ $\mathrm{H}, 0,-1.9099687975,-1.0956776417,-1.6684998694$ $\mathrm{C}, 0,-1.0593726622,0.067912648,1.4972712412$

$\mathrm{H}, 0,-1.9775388663,-0.2394897901,1.9906737906$ $\mathrm{H}, 0,-0.758509522,1.0233864957,1.9083684128$ $\mathrm{H}, 0,-0.2963536284,-0.6708342739,1.6861069307$ C, $0,-2.2870146183,1.2977582181,-0.3681554484$ $\mathrm{H}, \mathrm{O},-3.2590487066,1.0896820398,0.0687579019$ $\mathrm{H}, 0,-2.4018062518,1.3755954022,-1.4418501789$ $\mathrm{H}, 0,-1.9379052149,2.2481857121,0.0175880001$

Sum of electronic and zero-point Energies= Sum of electronic and thermal Energies= Sum of electronic and thermal Enthalpies= Sum of electronic and thermal Free Energies=

$-345.807496$ $-345.797519$ $-345.796575$ $-345.841977$

$-350.084760$ $-350.076010$ $-350.075066$ $-350.118927$

Dichlorocarbene (3), NImag $=0, C 2 \mathrm{~V}$

C, $0,0 ., 0 ., 0.8619667373$

$\mathrm{Cl}, 0,-1.42976095,0 .,-0.1521117772$

$\mathrm{Cl}, 0,1.42976095,0 \ldots,-0.1521117772$

Sum of electronic and zero-point Energies= Sum of electronic and thermal Energies= Sum of electronic and thermal Enthalpies= Sum of electronic and thermal Free Energies=

$-958.379094$

$-958.375654$

$-958.374710$

$-958.404876$

Insertion of 3 into acetonitrile, NImag=1, $-889.9 \mathrm{~cm}^{-1}$ C, $0,1.6834109945,-0.7294247424,0.7764645621$ $\mathrm{C}, 0,-0.4111751667,0.7277629343,0.7077281197$ $\mathrm{H}, 0,0.5084762063,0.1528222521,1.2100194881$ $\mathrm{H}, 0,1.9092527308,-1.0714965164,1.7876695168$ $\mathrm{Cl}, 0,-1.5804169167,-0.329548929,0.0233433957$ $\mathrm{Cl}, 0,0.0692136665,2.0299338542,-0.3435714644$ C, $0,1.2595472322,-1.7311800328,-0.1245178479$ $\mathrm{N}, 0,0.8135737511,-2.5131685204,-0.8704280266$ 
Sum of electronic and zero-point Energies=

$-1091.063193$

Sum of electronic and thermal Energies=

Sum of electronic and thermal Enthalpies=

$-1091.055980$

Sum of electronic and thermal Free Energies=

$-1091.055036$

$-1091.096574$

Insertion of 3 into methane, NImag=1, $-1144.9 \mathrm{~cm}^{-1}$ C, $0,-2.0945031133,-0.0000339127,-0.0397601078$ $\mathrm{C}, 0,0.0772558828,0.0000576018,-0.67486943$ $\mathrm{H}, 0,-1.0433555271,0.0000923247,-1.0244370067$ $\mathrm{H}, \mathrm{O},-2.5850032614,0.9195428571,-0.3606560432$ $\mathrm{H}, 0$, $-2.5848899913,-0.9196841701,-0.3606189069$ $\mathrm{H}, 0,-1.8118379151,0.0000081941,1.0079749953$ $\mathrm{Cl}, 0,0.5919808807,1.4685417185,0.1479431824$ $\mathrm{Cl}, 0,0.5920526533,-1.4685476797,0.1476752992$

Sum of electronic and zero-point Energies= Sum of electronic and thermal Energies= Sum of electronic and thermal Enthalpies= Sum of electronic and thermal Free Energies=

$-998.817005$

$-998.811376$

$-998.810432$

$-998.846809$

Insertion of 3 into isobutane, NImag=1, $-793.1 \mathrm{~cm}^{-1}$ C, $0,-1.4093254035,0.0327637192,0.3148011582$ C, $0,0.7560628988,0.0160377053,-0.9273871732$ $\mathrm{H}, 0,-0.3720219799,0.0530256492,-0.9199915637$ C, $0,-2.0236765329,1.3553064495,-0.070832194$ $\mathrm{H}, 0,-1.3503500897,2.190819896,0.1485062583$ $\mathrm{H}, 0,-2.9460070113,1.5191849956,0.5099563746$ $\mathrm{H}, 0,-2.2973735476,1.3913131544,-1.1315683259$ C, $0,-2.1092560737,-1.2061862645,-0.1856070981$ $\mathrm{H}, \mathrm{O},-3.0425386896,-1.359169462,0.3804758202$ $\mathrm{H}, 0,-1.4939565496,-2.101000077,-0.0432738536$ $\mathrm{H}, 0,-2.381036274,-1.1291706405,-1.2446591113$ C, $0,-0.8484433709,-0.0480006623,1.7019072586$ $\mathrm{H}, 0,-0.2740060221,-0.9675814037,1.8577683859$ $\mathrm{H}, 0,-1.6742342442,-0.0515115857,2.4327586705$ $\mathrm{H}, 0,-0.2122218258,0.8123655822,1.9363255197$ $\mathrm{Cl}, 0,1.4176555283,-1.503481773,-0.3274914424$ $\mathrm{Cl}, 0,1.514790185,1.4294934323,-0.1974256096$

Sum of electronic and zero-point Energies= Sum of electronic and thermal Energies= Sum of electronic and thermal Enthalpies= Sum of electronic and thermal Free Energies=

$-1116.688533$

$-1116.678588$

$-1116.677644$

$-1116.723886$

Cyclopentadienylidene (4), NImag $=0$

$\mathrm{H}, \mathrm{O},-2.2100004563,-1.0055296177,-0.0213193381$

C, $0,-1.1832446623,-0.6936990189,-0.1477600814$

C, $0,-0.7143479797,0.6281613171,-0.2088047573$

C, $0,0.7506661277,0.7047918012,-0.1465128996$

C, $0,1.2094297958,-0.5707577509,0.0524396635$

C, $0,-0.0216686639,-1.209003003,0.4120498037$

$\mathrm{H}, 0,-1.3485764246,1.477369112,0.0433110029$

$\mathrm{H}, 0,1.3061666153,1.6360437878,-0.1028599848$

$\mathrm{H}, 0,2.21157565,-0.9673766275,0.119456591$

Sum of electronic and zero-point Energies=

$-192.691713$

Sum of electronic and thermal Energies=

$-192.687467$ 
Insertion of 4 into acetonitrile, frozen atoms C, $-1,-1.7892428755,-0.806860704,0.1506997207$ $\mathrm{H},-1,-0.9486140335,-0.684029363,-0.6376756293$ $\mathrm{H}, 0,-2.3405325069,-1.6876895998,-0.1932320317$ C, $0,2.5484539646,-0.6185244404,0.195245779$ $\mathrm{H}, 0,3.5677348358,-0.9925025004,0.2269188489$ $\mathrm{C}, 0,-2.6127501032,0.3867488373,0.1399672605$ $\mathrm{N}, 0,-3.2594270984,1.351509373,0.1168833693$ $\mathrm{H}, 0,-1.359977317,-0.9953105621,1.1355147998$ C, $0,0.8753971095,0.9469524185,-0.1425361075$ $\mathrm{H}, 0,0.2927565888,1.8475381865,-0.274999986$ C, $0,1.4267829921,-1.3710959914,-0.0765718468$ $\mathrm{H}, 0,1.3591638836,-2.4426590832,-0.2022355182$ C, $-1,0.5630802515,-0.370653506,-0.6150922443$ C, $0,2.2059760642,0.8137506049,0.1663739477$ $\mathrm{H}, 0,2.9392738178,1.6121839961,0.2190068752$

Sum of electronic and zero-point Energies= Sum of electronic and thermal Energies= Sum of electronic and thermal Enthalpies= Sum of electronic and thermal Free Energies=

$-325.401631$

$-325.393446$

$-325.392502$

$-325.435825$

Insertion of 4 into acetonitrile, NImag=1, $-879.2 \mathrm{~cm}^{-1}, \mathrm{HF} / 3-21 \mathrm{G}$ * C, $0,0.2961381491,-0.4537625563,0.8890010095$ $\mathrm{C}, 0,0.9403924803,0.7668690581,0.3466581202$ $\mathrm{H}, \mathrm{O}, 0.586258769,1.7607564371,0.498435361$ $\mathrm{H}, 0,-1.0359366542,-0.4937884458,0.8164379519$ C, $0,-1.5915910834,-0.4269626047,-0.2784182044$ C, $0,1.012926345,-1.5969076848,0.2725473709$ $\mathrm{H}, 0,0.7350193767,-2.6211069179,0.3775880562$ C, $0,2.1093058208,0.3813601228,-0.1841941241$ $\mathrm{H}, 0,2.9082792917,1.0151567652,-0.5021067141$ C, $0,2.156007668,-1.1058937308,-0.2282341361$ $\mathrm{H}, 0,2.9961985698,-1.6683432944,-0.5739959462$ $\mathrm{H}, 0,-0.9765581345,-0.5970012519,-1.1434679421$ $\mathrm{H}, \mathrm{O},-2.3115791974,-1.2265113217,-0.1581146985$ C, $0,-2.2287309417,0.8661700638,-0.3131433569$ $\mathrm{N}, 0,-2.7240532355,1.8922288602,-0.3342965919$

Sum of electronic and zero-point Energies= Sum of electronic and thermal Energies= Sum of electronic and thermal Enthalpies= Sum of electronic and thermal Free Energies=

$-321.463310$ $-321.456335$ $-321.455391$ $-321.495591$

B3LYP / 6 - 31G (d) / / HF / $3-21 \mathrm{G}$ *

Sum of electronic and zero-point Energies= Sum of electronic and thermal Energies= Sum of electronic and thermal Enthalpies= Sum of electronic and thermal Free Energies=
$-325.396376$

$-325.389467$

$-325.426830$
$-325.390411$

Insertion of 4 into methane, NImag=1, $-80.8 \mathrm{~cm}^{-1}$ $\mathrm{C}, 0,0.1810526293,-0.000622845,-0.6084388902$ C, $0,-0.3693608922,1.1941192759,-0.0661283439$ $\mathrm{H}, 0,-0.0397195618,2.2116126649,-0.2180193335$ $\mathrm{H}, 0,1.9208159059,-0.0000696217,-0.6418512444$ $\mathrm{C}, 0,2.7931779083,0.0006843971,0.0676671325$ C, $0,-0.3687813414,-1.1943797119,-0.063262193$ 
$\mathrm{H}, \mathrm{O},-0.0384425614,-2.21203389,-0.2125326883$ C, $0,-1.6183786677,0.7348902712,0.2952332934$ $\mathrm{H}, 0,-2.5220540392,1.334284964,0.3597940181$ C, $0,-1.6180142231,-0.7349962891,0.2970483959$ $\mathrm{H}, 0,-2.5213568341,-1.3346982737,0.3633265238$ $\mathrm{H}, 0,3.3760885531,0.9005300838,-0.1435151242$ $\mathrm{H}, 0,2.4499020322,0.0013435564,1.1022842251$ $\mathrm{H}, 0,3.3765940266,-0.8991400726,-0.1422027451$

Sum of electronic and zero-point Energies= Sum of electronic and thermal Energies= Sum of electronic and thermal Enthalpies= Sum of electronic and thermal Free Energies=

$-233.165399$ $-233.158011$ $-233.157067$ $-233.197330$

Insertion of 4 into isobutane, frozen atoms C, $-1,0.6957031505,0.0970558559,0.8766628884$ C, $0,1.4023681078,1.1990238763,0.3153634688$ $\mathrm{H}, 0,1.0914966386,2.2334836791,0.2709911471$ $\mathrm{H},-1,-0.8323779825,0.1133522049,0.6592066604$ C, $-1,-1.5494368185,0.0133462229,-0.2462587996$ C, $0,-2.4046266789,-1.190151864,0.1655855142$ $\mathrm{H}, 0,-2.847993967,-1.0516335467,1.1575992231$ $\mathrm{H}, 0,-1.8137605309,-2.1120743915,0.1775452763$ $\mathrm{H}, 0,-3.2222750972,-1.3260669047,-0.5549852278$ C, $0,-2.3256098109,1.3331633462,-0.1765628306$ $\mathrm{H}, \mathrm{O},-2.7680415484,1.4912198649,0.8129454749$ $\mathrm{H}, 0,-3.1396085844,1.3222408046,-0.9138070084$ $\mathrm{H}, 0,-1.6792866017,2.1869268218,-0.4057838871$ C, $0,-0.8987703889,-0.192206436,-1.6114888215$ $\mathrm{H}, 0,-0.2478669888,0.6430876714,-1.8821315701$ $\mathrm{H}, 0,-1.6906255025,-0.2696347802,-2.3696752558$ $\mathrm{H}, 0,-0.3036966189,-1.1083960727,-1.6450333751$ C, $0,1.3319699218,-1.1536857962,0.6324044255$ $\mathrm{H}, 0,0.9603289123,-2.1429272658,0.860444709$ $\mathrm{C}, 0,2.6777569564,0.6817308739,0.1912591186$ $\mathrm{H}, 0,3.5949147631,1.2606604835,0.1391675583$ C, $0,2.6345058423,-0.7635606884,0.3859788365$ $\mathrm{H}, 0,3.5156314194,-1.3885309121,0.4958534725$

Sum of electronic and zero-point Energies= Sum of electronic and thermal Energies= Sum of electronic and thermal Enthalpies= Sum of electronic and thermal Free Energies=

$-351.023721$ $-351.013290$ $-351.012345$ $-351.060249$

Insertion of 4 into isobutane, NImag=1, $-421.9 \mathrm{~cm}^{-1}, \mathrm{HF} / 3-21 \mathrm{G} *$ C, $0,0.6662570289,0.1032636111,0.9330659687$

C, $0,1.3876250055,1.1731701298,0.2391075882$ $\mathrm{H}, 0,1.1046251486,2.2012792433,0.2174443362$ $\mathrm{H}, 0,-0.6124316675,0.1160214801,0.710231076$ C, $0,-1.4703533946,0.034105213,-0.1116749523$ C, $0,-2.27692848,-1.1495183293,0.4245918901$ $\mathrm{H}, 0,-2.5416815828,-1.0004179667,1.4638788533$ $\mathrm{H}, 0,-1.7191677639,-2.0727808311,0.3333134623$ $\mathrm{H}, 0,-3.1910389191,-1.2433855296,-0.1547080592$ $\mathrm{C}, 0,-2.1930310364,1.3673993469,0.0862599617$ $\mathrm{H}, 0,-2.4577918179,1.5153946499,1.1257028179$ $\mathrm{H}, 0,-3.104118172,1.3647070428,-0.5052726919$ $\mathrm{H}, 0,-1.5769035423,2.1953436597,-0.2404497077$ C, $0,-0.9832319772,-0.174036182,-1.5392690845$ $\mathrm{H}, \mathrm{0},-0.358588413,0.646418712,-1.8616603855$ $\mathrm{H}, \mathrm{0},-1.849653013,-0.2331605486,-2.1931407051$ 
$\mathrm{H}, 0,-0.4162734737,-1.0894047141,-1.6282584407$

C, $0,1.3097845723,-1.1567928817,0.5525622402$

$\mathrm{H}, 0,0.9598149061,-2.1333025846,0.8005850199$

C, $0,2.5565460891,0.635117012,-0.1713168918$

$\mathrm{H}, 0,3.4125293036,1.1672314683,-0.5272503901$

C, $0,2.5077982865,-0.8240085191,0.0249846585$

$\mathrm{H}, 0,3.3238824422,-1.4861404853,-0.1702834581$

Sum of electronic and zero-point Energies=

Sum of electronic and thermal Energies=

Sum of electronic and thermal Enthalpies=

Sum of electronic and thermal Free Energies=

$-346.623400$

$-346.613799$

$-346.612854$

$-346.658719$

B3LYP / 6 - 31G (d) // HF / $3-21 \mathrm{G} *$

Sum of electronic and zero-point Energies=

Sum of electronic and thermal Energies=

Sum of electronic and thermal Enthalpies=

Sum of electronic and thermal Free Energies=

$-351.025220$

$-351.016886$

$-351.015942$

$-351.059408$

Methylene (5), NImag $=0, C 2 \mathrm{v}$

C, $0,0.179232,0,0$.

$\mathrm{H}, 0,-0.5377,0.0 .859399$

$\mathrm{H}, \mathrm{O},-0.5377,0 .,-0.859399$

Sum of electronic and zero-point Energies=

Sum of electronic and thermal Energies=

Sum of electronic and thermal Enthalpies=

$-39.111654$

$-39.108815$

$-39.107871$

Sum of electronic and thermal Free Energies=

$-39.129361$

Insertion of 5 into acetonitrile, frozen atoms C, $-1,0.0024416606,-0.8708093696,0.084627902$

$\mathrm{H},-1,0.8817252136,-0.5305070876,-0.589416328$

$\mathrm{H}, \mathrm{O},-0.3203061264,-1.7881567172,-0.4181463184$

$\mathrm{C}, 0,-1.0356424,0.1423002615,0.0804301476$

$\mathrm{N}, 0,-1.8600025412,0.9602354674,0.0759771811$

$\mathrm{H}, 0,0.3290067493,-1.1069143129,1.0983337304$

C, $-1,2.2734583936,0.1013660694,-0.37089134$

$\mathrm{H}, 0,1.9804094328,1.0147994473,0.1915229152$

$\mathrm{H}, \mathrm{0}, 2.707636593,-0.5480113688,0.4208654758$

Sum of electronic and zero-point Energies= Sum of electronic and thermal Energies= Sum of electronic and thermal Enthalpies= Sum of electronic and thermal Free Energies=

$-171.827674$

$-171.822242$

$-171.821298$

$-171.855880$

Insertion of 5 into acetonitrile, NImag=1, $-1170.8 \mathrm{~cm}^{-1}, \mathrm{HF} / 3-21 \mathrm{G}$ * $\mathrm{C}, 0,0.3721130904,-0.0698063112,-0.7866865288$

C, $0,0.4801511473,-1.9620389048,0.3301800112$

$\mathrm{H}, 0,1.5075334494,-2.1381300123,0.026871193$

$\mathrm{H}, 0,0.4393519797,-1.4292463577,1.2753739656$

$\mathrm{H}, 0,-0.1053953321,-1.2141282711,-0.5685465786$

$\mathrm{H}, 0,0.0750772023,-0.0205893955,-1.8279673832$

$\mathrm{H}, 0,1.4368097205,0.0690920186,-0.7137265524$

$\mathrm{C}, 0,-0.3444049976,0.8960639802,0.0065127577$

$\mathrm{N}, 0,-0.91436178,1.649669919,0.6439939879$

Sum of electronic and zero-point Energies= Sum of electronic and thermal Energies= Sum of electronic and thermal Enthalpies= Sum of electronic and thermal Free Energies=

$-169.756617$

$-169.751832$

$-169.750888$

$-169.783915$ 
B3LYP / $6-31 \mathrm{G}(\mathrm{d}) / / \mathrm{HF} / 3-21 \mathrm{G}$ *

Sum of electronic and zero-point Energies=

$-171.834453$

Sum of electronic and thermal Energies=

Sum of electronic and thermal Enthalpies=

$-171.830262$

sum of electronic and thermal Free Energies=

$-171.829318$

$-171.861077$

Insertion of 5 into methane, frozen atoms C, $-1,-1.2422725926,0.0299515836,-0.6375108597$ $\mathrm{H},-1,0.2785322774,0.2121216436,-0.4442443497$ C, $-1,1.0425062274,0.0225833236,0.4069173703$ $\mathrm{H}, \mathrm{O},-1.6002641922,0.567279879,0.2695094019$ $\mathrm{H}, 0,-1.3315345595,-1.0355012502,-0.3270229394$ $\mathrm{H}, 0,1.7640329012,-0.6736775185,-0.0247476542$ $\mathrm{H}, 0,1.4870533897,1.0013578267,0.5968360665$ $\mathrm{H}, 0,0.6007783748,-0.3867900236,1.3132304116$

Sum of electronic and zero-point Energies= Sum of electronic and thermal Energies= Sum of electronic and thermal Enthalpies= Sum of electronic and thermal Free Energies=

$-79.604849$

$-79.600278$

$-79.599334$

$-79.630146$

Insertion of 5 into methane, NImag=1, $-1082.1 \mathrm{~cm}^{-1}, \mathrm{HF} / 3-21 \mathrm{G}$ * C, $0,1.1679518113,0.0004285972,-0.2474839014$ $\mathrm{H}, \mathrm{O},-0.0270958935,0.025129523,-0.7147517328$ C, $0,-0.9632617628,0.002746057,0.1153255009$ $\mathrm{H}, 0,1.2848103467,-0.9144607386,0.3257842792$ $\mathrm{H}, 0,1.2974133914,0.8713753951,0.3881264023$ $\mathrm{H}, \mathrm{O},-1.4819839448,0.9095839476,-0.16016346$ $\mathrm{H}, 0,-1.494935113,-0.8748114841,-0.2231255986$ $\mathrm{H}, 0,-0.806349078,-0.0358645684,1.1770805128$

Sum of electronic and zero-point Energies= Sum of electronic and thermal Energies= Sum of electronic and thermal Enthalpies= Sum of electronic and thermal Free Energies=

$-78.543296$

$-78.539325$

$-78.538381$

$-78.567671$

B3LYP / 6 - 31G (d) / / HF / 3 - 21G*

Sum of electronic and zero-point Energies= Sum of electronic and thermal Energies= Sum of electronic and thermal Enthalpies= Sum of electronic and thermal Free Energies=
$-79.599784$

$-79.596415$

$-79.595471$

$-79.623160$

Insertion of 5 into isobutane, frozen atoms C, $-1,-2.0675567491,0.0423212996,-1.0501710282$ $\mathrm{H},-1,-0.5467518841,0.2244913556,-0.8569045172$ C, $-1,0.2172220659,0.0349530386,-0.0057428012$ C, $0,0.6787135065,-1.4148450777,-0.1598764147$ $\mathrm{H}, 0,0.9550768364,-1.6371321957,-1.1960218923$ $\mathrm{H}, 0,-0.1004541189,-2.1215353503,0.1438661844$ $\mathrm{H}, 0,1.5597603455,-1.5953656755,0.4706063132$ C, $0,1.3069503799,1.0307595136,-0.4354554377$ $\mathrm{H}, 0,1.6446903454,0.8381925131,-1.4591683477$ $\mathrm{H}, 0,2.1747038418,0.941903926,0.2321448839$ $\mathrm{H}, 0,0.9452930028,2.0632533776,-0.3830997774$ $\mathrm{C}, 0,-0.2812227768,0.3425321581,1.4058925217$ $\mathrm{H}, 0,-0.6767226484,1.3607918683,1.4839700097$ $\mathrm{H}, 0,0.5578507807,0.2597789472,2.1113818677$ $\mathrm{H}, \mathrm{O},-1.059950017,-0.3542245188,1.7298556841$ $\mathrm{H}, \mathrm{O},-2.4004653612,0.7482552383,-0.2580424068$ 
$\mathrm{H}, 0,-2.1776696814,-0.9427350784,-0.546469041$

Sum of electronic and zero-point Energies=

$-197.449703$

Sum of electronic and thermal Energies=

Sum of electronic and thermal Enthalpies=

$-197.441226$

Sum of electronic and thermal Free Energies=

$-197.479995$

Insertion of 5 into isobutane, NImag=1, $-887.4 \mathrm{~cm}^{-1}, \mathrm{HF} / 3-21 \mathrm{G}$ * $\mathrm{C}, 0,-0.0613271761,0.1035402334,-0.0868374532$

C, $0,-0.1733633222,-1.8506782198,0.9444387506$

$\mathrm{H}, 0,0.7184820379,-2.2100016083,0.4377178347$

$\mathrm{H}, 0,0.0988193727,-1.3848017852,1.8880416829$

$\mathrm{H}, 0,-0.6483547192,-0.950502681,0.2269035928$

C, $0,-0.3217073341,0.029137827,-1.5985899772$

$\mathrm{H}, 0,0.2850091374,-0.7431838226,-2.0567729926$

$\mathrm{H}, 0,-0.0675046321,0.9818716783,-2.0539405053$

$\mathrm{H}, 0,-1.3635703349,-0.1843479254,-1.8057603462$

C, $0,1.411041475,0.4031004737,0.1985892359$

$\mathrm{H}, 0,2.0622567244,-0.3335955835,-0.2537394764$

$\mathrm{H}, 0,1.6123703546,0.439127578,1.2610901954$

$\mathrm{H}, 0,1.6557170823,1.3749022889,-0.2257187973$

C, $0,-0.9790512052,1.1247962655,0.5936268895$

$\mathrm{H}, 0,-0.8176318522,1.1376620641,1.6651071503$

$\mathrm{H}, 0,-2.0219516043,0.8976383474,0.4069080227$

$\mathrm{H}, 0,-0.7671961912,2.1158519696,0.2027989655$

Sum of electronic and zero-point Energies= Sum of electronic and thermal Energies= Sum of electronic and thermal Enthalpies=

Sum of electronic and thermal Free Energies=

$-194.911320$

$-194.904117$

$-194.903172$

$-194.942348$

$-197.459064$

$-197.453609$

$-197.452664$

$-197.487494$

Difluorocarbene (6), NImag $=0, C 2 \mathrm{~V}$

$\mathrm{C}, 0,-0.247366061,-0.4284509115,-0.3498288665$

$\mathrm{F}, 0,-0.8142046634,0.6605038496,0.1166091683$

$\mathrm{F}, 0,0.9791153707,-0.3748699086,0.116610076$

Sum of electronic and zero-point Energies=

Sum of electronic and thermal Energies=

Sum of electronic and thermal Enthalpies=

Sum of electronic and thermal Free Energies=

$-237.682800$

$-237.679802$

$-237.678858$

$-237.706221$

Insertion of 6 into acetonitrile, NImag=1, $-999.9 \mathrm{~cm}^{-1}$ $\mathrm{C}, 0,0.7989987838,1.2654106686,-0.1247860802$ $\mathrm{H}, 0,0.4587569909,1.805300656,0.7573818341$ C, $0,-1.2434833764,0.0600362177,-0.4641423307$ $\mathrm{H}, 0,-0.6055371535,0.8638662022,-0.9423353814$ C, $0,1.6256574635,0.1437637273,0.1166390905$ $\mathrm{N}, 0,2.2266578947,-0.8426745002,0.2909952941$ $\mathrm{F}, 0,-0.7939005094,-1.1676782832,-0.4644607934$ $\mathrm{F}, 0,-1.8330604381,0.3344888041,0.6784056062$ $\mathrm{H}, 0,1.1157862022,1.9129962729,-0.9437809049$

Sum of electronic and zero-point Energies= Sum of electronic and thermal Energies= 
Insertion of 6 into methane, NImag=1, $-1336.7 \mathrm{~cm}^{-1}$ C, $0,-1.6669883789,0.0000597509,0.1042869421$ C, $0,0.3977245211,0.0000658537,-0.5215995511$ $\mathrm{H}, 0,-0.6227883318,0.0002186307,-1.0219078031$ $\mathrm{F}, 0,0.7752456625,1.0862369072,0.1517632463$ $\mathrm{F}, 0,0.7750621532,-1.0863542799,0.151467152$ $\mathrm{H}, \mathrm{O},-2.1923412851,0.915506813,-0.1711075095$ $\mathrm{H}, 0,-2.1919721623,-0.9156536544,-0.1709228642$ $\mathrm{H}, 0,-1.3300854145,0.0002309368,1.1387402462$

Sum of electronic and zero-point Energies= Sum of electronic and thermal Energies= Sum of electronic and thermal Enthalpies= Sum of electronic and thermal Free Energies=
$-278.095601$

$-278.090487$

$-278.089543$

$-278.123549$

Insertion of 6 into isobutane, NImag=1, $-1093.3 \mathrm{~cm}^{-1}$ C, $0,-0.8266834744,-0.0040413153,0.3014654544$ C, $0,0.9959242826,0.0022972009,-1.0530127316$ $\mathrm{H}, \mathrm{O},-0.1041236992,-0.0053693128,-1.1716979124$ $\mathrm{F}, 0,1.5619350537,1.1023453791,-0.5354298731$ $\mathrm{F}, 0,1.5759583682,-1.0866698516,-0.5275232029$ C, $0,-1.5787005425,1.2771197306,0.016322877$ $\mathrm{H}, \mathrm{O},-2.3359022647,1.4719365187,0.7923111792$ $\mathrm{H}, 0,-2.1044452641,1.2443523449,-0.9462771583$ $\mathrm{H}, 0,-0.8965632966,2.1361452479,0.0080613511$ C, $0,-1.562920535,-1.2965076851,0.0261347697$ $\mathrm{H}, 0,-2.3180679723,-1.494438357,0.8033371434$ $\mathrm{H}, 0,-0.8703638043,-2.1471944716,0.0248965078$ $\mathrm{H}, \mathrm{O},-2.0885859782,-1.2777309157,-0.9368888852$ $\mathrm{C}, 0,-0.0315684871,0.0056233247,1.5800375477$ $\mathrm{H}, \mathrm{O},-0.7080337666,0.0044018368,2.4492833581$ $\mathrm{H}, 0,0.5987277011,0.8982014168,1.6557772009$ $\mathrm{H}, 0,0.6100100864,-0.8783315902,1.662087397$

Sum of electronic and zero-point Energies= Sum of electronic and thermal Energies= Sum of electronic and thermal Enthalpies= Sum of electronic and thermal Free Energies=

$-395.964793$

$-395.955609$

$-395.954665$

$-395.998561$

Dimethylamino (trimethylphosphonio) carbene 7, NImag=0 P , 0, - $1.0162606545,0.0256254868,0.154437419$ C, $0,-2.043113381,-0.1825761833,-1.3381301324$ $\mathrm{H}, \mathrm{O},-3.0847193731,0.0535918527,-1.099725349$ $\mathrm{H}, 0,-1.9692537529,-1.2134960694,-1.6910079159$ $\mathrm{H}, 0,-1.6998550906,0.4922827115,-2.1282226726$ $\mathrm{C}, 0,-1.32240351,1.7454405582,0.7523679063$ $\mathrm{H}, \mathrm{O},-2.3936496726,1.8706886898,0.9470452626$ $\mathrm{H}, 0,-1.0201099322,2.4775557951,-0.0020963999$ $\mathrm{H}, 0,-0.7775572728,1.9352881464,1.681720145$ C, $0,-1.641185637,-1.0836832728,1.4599681734$ $\mathrm{H}, 0,-1.5643980976,-2.1205091868,1.1255072049$ $\mathrm{H}, 0,-2.6861358437,-0.8404180025,1.6755035757$ $\mathrm{H}, 0,-1.0533189603,-0.9574285654,2.3742898417$ C, $0,0.5420789376,-0.7551650596,-0.3209076518$ $\mathrm{N}, 0,1.7190335599,-0.2209218714,-0.3183108294$ C, $0,2.1489461632,1.1477343856,0.0602480252$ 
$\mathrm{H}, 0,1.294013428,1.7409983412,0.3756947767$

$\mathrm{H}, 0,2.6311576882,1.6207274109,-0.8001417956$

$\mathrm{H}, 0,2.8747248674,1.080664135,0.8758791707$

C, $0,2.8592681212,-1.0684173018,-0.7550521701$

$\mathrm{H}, 0,2.4741239345,-2.0514418851,-1.0172454823$

$\mathrm{H}, 0,3.5823355227,-1.1446110023,0.06246431$

$\mathrm{H}, 0,3.3417732886,-0.6018203316,-1.6190150547$

Sum of electronic and zero-point Energies=

Sum of electronic and thermal Energies=

Sum of electronic and thermal Enthalpies=

Sum of electronic and thermal Free Energies=

$-633.297067$

$-633.284880$

$-633.283936$

$-633.334260$

Insertion of 7 into acetonitrile, NImag=1, $-976.7 \mathrm{~cm}^{-1}$ C, $0,1.2420985115,1.9551325645,-0.9523381149$ $\mathrm{H}, 0,0.5903934209,2.7473175424,-0.5840033291$ C, $0,0.0327714152,-0.337545931,-0.7655692488$ $\mathrm{H}, 0,0.4507477789,0.639178857,-1.3559476686$ $\mathrm{C}, 0,2.189339004,1.463878538,-0.0224050771$ $\mathrm{N}, 0,2.8626585048,0.8720113061,0.7283243187$ $\mathrm{H}, 0,1.6325846064,2.1650638514,-1.9495905956$ $\mathrm{N}, 0,0.9321769128,-1.20127087,-0.3363115725$ $\mathrm{P}, 0,-1.4470936203,0.1451659885,0.1623905186$ C, $0,1.9500423131,-1.6795726394,-1.2940455815$ $\mathrm{H}, 0,1.8012321323,-1.1865808605,-2.2541827386$ $\mathrm{H}, 0,1.8372052325,-2.7616434335,-1.423477317$ $\mathrm{H}, 0,2.9447821464,-1.4570522294,-0.9020994947$ C, $0,1.0369527648,-1.8229836249,0.9959111952$ $\mathrm{H}, 0,2.0729312746,-1.7720334685,1.3340145479$ $\mathrm{H}, 0,0.7262346655,-2.8719396129,0.9341682204$ $\mathrm{H}, 0,0.4174341602,-1.2935924951,1.7175369502$ C, $0,-2.4765135368,-1.3475414333,0.3627515492$ $\mathrm{H}, \mathrm{O},-2.6092596199,-1.8361839522,-0.6066053439$ $\mathrm{H}, 0,-3.4569460901,-1.0601469938,0.7551460996$ $\mathrm{H}, 0,-2.0111199319,-2.0507265588,1.0584961325$ C, $0,-1.2866100239,0.956315514,1.799334854$ $\mathrm{H}, \mathrm{O},-2.2705687387,1.3201532399,2.1149756223$ $\mathrm{H}, 0,-0.5962012125,1.8011199252,1.7272216883$ $\mathrm{H}, 0,-0.9102081169,0.2603605928,2.5528742284$ C, $0,-2.3387086467,1.2958128481,-0.9305941992$ $\mathrm{H}, 0,-2.4867973049,0.8298542302,-1.9083869135$ $\mathrm{H}, 0,-1.7737269082,2.2218901719,-1.0576637384$ $\mathrm{H}, 0,-3.3123919207,1.5313132974,-0.490695614$

Sum of electronic and zero-point Energies= Sum of electronic and thermal Energies= Sum of electronic and thermal Enthalpies= Sum of electronic and thermal Free Energies=

$-765.967947$

$-765.951426$

$-765.950482$

$-766.011608$

Insertion of 7 into methane, NImag=1, $-1201.7 \mathrm{~cm}^{-1}$ C, $0,-2.4221573109,-0.6994991844,0.1009321393$ $\mathrm{C}, 0,-0.4890031338,0.372512076,-0.4867194739$ $\mathrm{H}, 0,-1.4983154225,-0.123282274,-0.8796378272$ $\mathrm{N}, 0,-0.4770770535,1.6403685956,-0.0456895913$ $\mathrm{P}, 0,0.7089640539,-0.8732461317,-0.087055843$ C, $0,-1.1366000667,2.6529962571,-0.8814484833$ $\mathrm{H}, 0,-0.6183183662,3.6105131098,-0.7711771525$ $\mathrm{H}, \mathrm{O},-2.184492615,2.7900631173,-0.5807080394$ $\mathrm{H}, 0,-1.0959138352,2.3397120192,-1.9247689308$ C, $0,2.3060357595,-0.2404432748,-0.6991703834$ $\mathrm{H}, 0,2.2308039958,-0.0244351879,-1.7680186943$ 
$\mathrm{H}, 0,3.089414964,-0.9855839339,-0.5304147974$ $\mathrm{H}, 0,2.5693712607,0.6799810778,-0.1704152967$ C, $0,-0.3183374603,2.0393278009,1.3585981971$ $\mathrm{H}, 0,0.1813212438,3.0107127164,1.4108331999$ $\mathrm{H}, 0,0.2805946275,1.3069213099,1.900981394$ $\mathrm{H}, \mathrm{O},-1.2964738312,2.1239050634,1.8519643491$ C, $0,0.2685132566,-2.3718758785,-1.0245507854$ $\mathrm{H}, 0,0.0496892938,-2.107958552,-2.062317871$ $\mathrm{H}, 0,-0.5992803512,-2.8683709617,-0.5837544679$ $\mathrm{H}, 0,1.115397697,-3.0644644655,-1.0019140883$ C, $0,1.0060598007,-1.4206301416,1.6415224923$ $\mathrm{H}, 0,1.7033843359,-2.2654866708,1.6356808824$ $\mathrm{H}, 0,0.0703952698,-1.7414674682,2.1076579014$ $\mathrm{H}, 0,1.4432355592,-0.614897888,2.236978437$ $\mathrm{H}, \mathrm{O},-2.8806258803,-1.3633516824,-0.6354866936$ $\mathrm{H}, \mathrm{0},-3.0521981003,0.1535879257,0.3472557432$ $\mathrm{H}, \mathrm{O},-2.0899763505,-1.2343113775,0.98794452$

Sum of electronic and zero-point Energies= Sum of electronic and thermal Energies= Sum of electronic and thermal Enthalpies= Sum of electronic and thermal Free Energies=

$-673.721142$

$-673.706238$

$-673.705293$

$-673.761641$

Insertion of 7 into isobutane, NImag=1, $-699.1 \mathrm{~cm}^{-1}$ C, $0,-2.2040478565,-0.4805329737,-0.0530773748$ C, $0,0.1823122092,0.5205259676,-0.4060165275$ $\mathrm{H}, 0,-0.8196955436,0.0983221563,-0.7755123917$ C, $0,-2.9639754389,0.0570683183,-1.2458954972$ $\mathrm{H}, \mathrm{O},-2.8866975978,1.1461768257,-1.3295235781$ $\mathrm{H}, 0,-4.0335004366,-0.1809780494,-1.1281696025$ $\mathrm{H}, 0,-2.6377591416,-0.3934607695,-2.1896982702$ $\mathrm{C}, 0,-2.1557585117,-1.9824640604,0.0419330174$ $\mathrm{H}, \mathrm{O},-3.1656454476,-2.3561733306,0.2815346536$ $\mathrm{H}, 0,-1.4970212599,-2.3392407524,0.8405608762$ $\mathrm{H}, 0,-1.8759647454,-2.4556426915,-0.9054104156$ $\mathrm{N}, 0,0.1882313883,1.7369312181,0.2011630065$ $\mathrm{P}, 0,1.4212885961,-0.7066435683,-0.1750670268$ C, $0,-2.4025809474,0.2776938659,1.2229777205$ $\mathrm{H}, 0,-1.9609463136,-0.2303813246,2.0858878648$ $\mathrm{H}, 0,-3.4776763573,0.4095343309,1.4221210748$ $\mathrm{H}, \mathrm{O},-1.9768083172,1.2883502929,1.1541346755$ C, $0,-0.1826225173,2.9239505219,-0.5800905296$ $\mathrm{H}, 0,0.7007293395,3.5500810151,-0.7694098007$ $\mathrm{H}, 0,-0.9212388513,3.5255163555,-0.0385491099$ $\mathrm{H}, 0,-0.5968670737,2.6097917026,-1.5381047855$ C, $0,3.0319018679,0.083744456,-0.5251824837$ $\mathrm{H}, 0,3.0074047353,0.5451330807,-1.5154189887$ $\mathrm{H}, 0,3.8242436214,-0.6704993838,-0.4941725977$ $\mathrm{H}, 0,3.2516571179,0.8515473456,0.2214864172$ C, $0,0.7494386127,2.0291214332,1.5202302308$ $\mathrm{H}, 0,1.7855549336,2.3922361615,1.4500811461$ $\mathrm{H}, 0,0.7174116096,1.1387734969,2.1504026618$ $\mathrm{H}, 0,0.1627109371,2.8133347825,2.0099831795$ C, $0,1.1645898085,-2.0282677804,-1.4063346031$ $\mathrm{H}, 0,1.0137474346,-1.5874614893,-2.3945591747$ $\mathrm{H}, 0,0.2975621405,-2.638369673,-1.1447332142$ $\mathrm{H}, 0,2.0492061379,-2.6722529809,-1.428129574$ C, $0,1.6594528913,-1.5844370986,1.4292344449$ $\mathrm{H}, 0,2.4403168223,-2.3455457954,1.3233051846$ $\mathrm{H}, 0,0.7296307475,-2.073581541,1.7317934167$ $\mathrm{H}, 0,1.9604361416,-0.8824906657,2.2112943224$ 
Sum of electronic and zero-point Energies=

Sum of electronic and thermal Energies=

Sum of electronic and thermal Enthalpies=

791.584911

$-791.565755$

$-791.564811$

Sum of electronic and thermal Free Energies=

$-791.630518$

Benzocyclobutenylidene (8), NImag=0

$\mathrm{H}, \mathrm{O},-1.1209853997,-2.446737363,-0.2226472796$

C, $0,-0.4629152944,-1.584798544,-0.1588898625$

C, $0,1.3100626518,0.7014994005,0.0333705353$

C, $0,-0.9476300788,-0.2571485062,-0.0838354249$

C, $0,0.9119640044,-1.7237812736,-0.1365969756$

$\mathrm{C}, 0,1.781933567,-0.6032434607,-0.0064031831$

$\mathrm{C}, 0,-0.0746937191,0.8643562936,-0.1630591975$

$\mathrm{H}, 0,1.3532900667,-2.7159197432,-0.1872947163$

$\mathrm{H}, 0,2.8521399083,-0.7859276009,0.0466075893$

$\mathrm{H}, 0,1.9912620822,1.5453282199,0.1043236799$

C, $0,-1.7704944356,0.6281039009,0.6664878489$

C, $0,-1.1550277067,1.8883443894,-0.0393372749$

$\mathrm{H}, 0,-1.0037169659,2.7795897365,0.5765921967$

$\mathrm{H}, 0,-1.6311836233,2.14367355,-0.9880002653$

Sum of electronic and zero-point Energies= Sum of electronic and thermal Energies= Sum of electronic and thermal Enthalpies=

$-308.188457$

$-308.182627$

$-308.181683$

$-308.218041$

Insertion of 8 into acetonitrile, NImag=1, $-1008.9 \mathrm{~cm}^{-1}$

C, $0,2.4851981191,1.2721319585,-1.1518235322$

$\mathrm{H}, 0,2.4709651556,2.0623456096,-0.3986794459$

C, $0,0.084841203,0.5271354463,-1.2515056424$

$\mathrm{H}, 0,1.2220128181,1.149047413,-1.5286713901$

$\mathrm{C}, 0,3.0722993717,0.053636125,-0.6786828387$

$\mathrm{N}, 0,3.4899547214,-0.9678800762,-0.3017765709$

$\mathrm{H}, 0,2.924586971,1.6196090235,-2.0908361262$

C, $0,-0.0104720451,-1.0268121258,-1.3020730599$

$\mathrm{H}, 0,0.9109159103,-1.5387925507,-1.0021675289$

$\mathrm{H}, 0,-0.3766889584,-1.4551480048,-2.2424110803$

C, $0,-0.9808525175,-0.8097546474,-0.1493997127$

C, $0,-0.5540388954,0.5213332051,0.0459738453$

C, $0,-2.0609974945,-1.3451351659,0.5414777575$

$\mathrm{H}, \mathrm{O},-2.4487988756,-2.3434478941,0.3595193863$

C, $0,-1.086469381,1.3351412666,1.0609946421$

$\mathrm{H}, 0,-0.7373002376,2.3459496983,1.2527823711$

C, $0,-2.5882821589,-0.5372419983,1.5568697634$

C, $0,-2.1024086124,0.7639632772,1.8210890838$

$\mathrm{H}, 0,-3.3956798726,-0.922427851,2.1744607081$

$\mathrm{H}, \mathrm{0},-2.5526014939,1.331641042,2.6309172652$

Sum of electronic and zero-point Energies=

Sum of electronic and thermal Energies=

Sum of electronic and thermal Enthalpies=

Sum of electronic and thermal Free Energies=

$-440.893004$

$-440.883168$

$-440.882224$

$-440.929932$

Insertion of 8 into methane, NImag=1, $-860.5 \mathrm{~cm}^{-1}$ $\mathrm{H}, 0,0.033277257,-2.4905846927,-0.5544345701$

C, $0,-0.4421860122,-1.5536550597,-0.2762254409$

C, $0,-1.7448055093,0.9230357087,0.4479442802$

C, $0,0.2187830823,-0.3194177682,-0.3261832184$

C, $0,-1.7688192179,-1.518697111,0.1574623354$ 
C, $0,-2.4103139607,-0.3106314368,0.4972091696$

$C, 0,-0.4052692262,0.8732046916,0.0872250716$

$\mathrm{H}, 0,-2.3334915826,-2.4452047715,0.2258008624$

$\mathrm{H}, 0,-3.4494456283,-0.3428156101,0.8149871664$

$\mathrm{H}, 0,-2.2529545094,1.8439422834,0.7226271014$

C, $0,1.3362112443,0.4145547643,-0.9217916471$

$\mathrm{C}, 0,0.8396205004,1.7045407228,-0.1833037881$

$\mathrm{H}, 0,0.7486223882,2.5995277442,-0.8111083842$

$\mathrm{H}, 0,1.3974421866,1.967770414,0.7229375695$

$\mathrm{H}, 0,2.5419096476,0.0365246128,-0.8381914727$

C, $0,3.219835968,-0.2454605953,0.2402364749$

$\mathrm{H}, 0,4.0739721681,0.426345364,0.1330374918$

$\mathrm{H}, 0,3.4789755101,-1.2907201167,0.0622345485$

$\mathrm{H}, 0,2.7033513499,-0.1096287254,1.186670265$

Sum of electronic and zero-point Energies=

Sum of electronic and thermal Energies=

Sum of electronic and thermal Enthalpies=

Sum of electronic and thermal Free Energies=

$-348.646092$

$-348.637803$

$-348.636859$

$-348.679382$

Insertion of 8 into isobutane, NImag=1, $-589.5 \mathrm{~cm}^{-1}$ $\mathrm{H}, 0,0.9912412637,-0.4235806967,2.417976518$

C, $0,0.0059327511,-0.6184196629,2.0015574965$

C, $0,-2.6201011811,-1.1781967865,0.9303387512$

C, $0,-0.2405086256,-0.6800577725,0.6217953707$

$\mathrm{C}, 0,-1.096682836,-0.8325901904,2.8310061394$

C, $0,-2.3744486215,-1.1214909229,2.3113892365$

C, $0,-1.5390216458,-0.883804086,0.1125033137$

$\mathrm{H}, 0,-0.9709027189,-0.7906290394,3.9101712421$

$\mathrm{H}, 0,-3.1932712775,-1.2943767699,3.0053606474$

$\mathrm{H}, 0,-3.6153115011,-1.3920601288,0.548366861$

C, $0,0.3685081065,-0.8924830046,-0.689454703$

C, $0,-1.0610742171,-0.7723478172,-1.3271272957$

$\mathrm{H}, \mathrm{0},-1.3308223313,-1.5639001687,-2.0375737159$

$\mathrm{H}, 0,-1.2883148532,0.1981782296,-1.7852083962$

$\mathrm{H}, 0,1.2760201306,-0.102312742,-1.1029293052$

C, $0,1.6477737301,1.1316875868,-1.3118841734$

C, $0,3.017490455,1.0507651322,-0.6519240034$

$\mathrm{H}, 0,3.6069569601,0.2180261302,-1.0512632527$

$\mathrm{H}, 0,2.9292077718,0.9181480139,0.4323434642$

$\mathrm{H}, 0,3.5813081791,1.9787386467,-0.8285220414$

$\mathrm{C}, 0,1.6986924208,1.1650004927,-2.8309877223$

$\mathrm{H}, 0,2.2616094506,0.3141623192,-3.2311645923$

$\mathrm{H}, 0,2.1936128881,2.0852540731,-3.1759067037$

$\mathrm{H}, 0,0.6943383561,1.1471978824,-3.2683713314$

C, $0,0.7454345234,2.1820581229,-0.6984163227$

$\mathrm{H}, 0,-0.2561507056,2.1750373031,-1.1425549096$

$\mathrm{H}, 0,1.1686250012,3.1846331739,-0.8667709674$

$\mathrm{H}, \mathrm{0}, 0.6398842269,2.0467572233,0.3832699576$

Sum of electronic and zero-point Energies=

Sum of electronic and thermal Energies=

Sum of electronic and thermal Enthalpies=

Sum of electronic and thermal Free Energies=

$-466.510167$

$-466.497667$

$-466.496722$

$-466.549513$

Cyclopent-2-enylidene (9), NImag $=0$

C, $0,-0.5336367461,-1.1884136815,-0.4702797537$

C, $0,-1.2484996443,0.062698595,-0.2688954279$

C, $0,-0.4629082249,1.069692897,0.2058648421$

$\mathrm{H}, 0,-2.3098071529,0.1825985509,-0.4779685214$

$\mathrm{H}, \mathrm{O},-0.7963519122,2.0845968252,0.4250089413$ 
C, $0,0.8729280842,-0.8494889463,-0.0467554447$

$\mathrm{H}, 0,1.5520427218,-1.0762828488,-0.8823167467$

$\mathrm{H}, 0,1.1760443004,-1.5508367339,0.7451060277$

C, $0,0.9503629522,0.6315497416,0.4029306541$

$\mathrm{H}, 0,1.6431738282,1.2412922764,-0.1958327653$

$\mathrm{H}, 0,1.2654196881,0.762400295,1.4488138452$

Sum of electronic and zero-point Energies=

Sum of electronic and thermal Energies=

Sum of electronic and thermal Enthalpies=

$-193.911064$

$-193.906274$

Sum of electronic and thermal Free Energies=

$-193.905330$

$-193.938874$

Insertion of 9 into acetonitrile, NImag=1, $-1084.6 \mathrm{~cm}^{-1}$

C, $0,-2.2059225459,0.5951452558,0.4169289024$

$\mathrm{H}, \mathrm{O},-2.7385705234,1.4497164214,0.8433716505$

C, $0,0.3125224452,0.341244811,1.0667934934$

$\mathrm{H}, 0,-1.0245895606,0.704985547,0.992562085$

C, $0,-2.0597946347,0.6804906873,-1.0038024493$

$\mathrm{N}, 0,-1.8187865418,0.7554258351,-2.1436946922$

$\mathrm{H}, 0,-2.6429132495,-0.3493187254,0.7476755184$

C, $0,1.1618348643,0.6881720146,-0.1277358001$

$\mathrm{H}, 0,1.6492345085,1.6635051434,-0.0072094907$

$\mathrm{H}, 0,0.5039079153,0.7958585787,-1.0073997781$

C, $0,0.559373808,-1.0513123905,1.327478935$

$\mathrm{H}, 0,0.0153264206,-1.6333698436,2.0668860281$

C, $0,1.639440161,-1.5083218977,0.6322267891$

$\mathrm{H}, \mathrm{0}, 2.1108093446,-2.4797553635,0.7688839211$

C, $0,2.1641758723,-0.4796825201,-0.3171426085$

$\mathrm{H}, 0,3.1769589719,-0.1657738115,-0.0258837748$

$\mathrm{H}, 0,2.2515621439,-0.8682445549,-1.3415068863$

Sum of electronic and zero-point Energies=

Sum of electronic and thermal Energies=

Sum of electronic and thermal Enthalpies=

Sum of electronic and thermal Free Energies=

$-326.619764$

$-326.611431$

$-326.610487$

$-326.653729$

Insertion of 9 into methane, NImag=1, $-988.5 \mathrm{~cm}^{-1}$

C, $0,-2.5066839442,0.0141428591,0.2555645247$

C, $0,-0.4095306772,0.0149386477,-0.8559733769$

$\mathrm{H}, \mathrm{O},-1.6761520629,0.0522993352,-0.787012385$

C, $0,0.3539270158,1.1876733561,-0.2445059276$

$\mathrm{H}, 0,-0.2831530318,1.7809911575,0.4208953035$

$\mathrm{H}, 0,0.673851727,1.8726305427,-1.04031496$

C, $0,0.2018301724,-1.1761139179,-0.3084464937$

C, $0,1.3503773189,-0.9007961403,0.3579415623$

C, $0,1.576875806,0.5790911733,0.5015713536$

$\mathrm{H}, 0,2.5146451465,0.8885838903,0.0198891291$

$\mathrm{H}, 0,1.6689166481,0.8788045696,1.556764526$

$\mathrm{H}, 0,-0.1676373602,-2.1793919968,-0.507118462$

$\mathrm{H}, 0,2.0678005247,-1.6405827566,0.7062181041$

$\mathrm{H}, \mathrm{O},-3.0804469719,0.9306916875,0.0990167383$

$\mathrm{H}, \mathrm{O},-3.092804323,-0.8867585087,0.0638981153$

$\mathrm{H}, 0$, - $2.0257944474,-0.010883788,1.230854036$

Sum of electronic and zero-point Energies=

Sum of electronic and thermal Energies=

Sum of electronic and thermal Enthalpies=

Sum of electronic and thermal Free Energies=

$-234.366725$

$-234.359654$

$-234.358710$

$-234.398307$ 
Insertion of 9 into isobutane, NImag=1, $-732.1 \mathrm{~cm}^{-1}$ C, $0,-1.6390292945,0.0667846901,-0.0015215102$ C, $0,0.708437956,-0.0233235082,-0.924163821$ $\mathrm{H}, 0,-0.5761823525,0.0529414811,-0.7739144556$ C, $0,-1.3345667556,-0.0446196496,1.4778620266$ $\mathrm{H}, 0,-0.8021532694,-0.9718718903,1.7164716831$ $\mathrm{H}, 0$, - $2.2672559912,-0.036244467,2.0641235562$ $\mathrm{H}, 0,-0.727483504,0.7965338047,1.8344294326$ C, $0,-2.2351975238,1.4067639904,-0.4034636008$ $\mathrm{H}, \mathrm{0},-3.227549746,1.5445567221,0.0526467163$ $\mathrm{H}, 0,-2.3543709311,1.4815336061,-1.4905519267$ $\mathrm{H}, 0,-1.6050862614,2.241427316,-0.072686846$ C, $0,1.4860265209,1.1246353968,-0.2847596656$ $\mathrm{H}, 0,0.8727399784,1.6419487382,0.4665600716$ $\mathrm{H}, 0,1.7526430112,1.8863973564,-1.0274815701$ C, $0,1.2570551706,-1.2189874624,-0.3290095341$ C, $0,2.4267373801,-0.9831045145,0.3233789636$ $\mathrm{C}, 0,2.7436571835,0.4841856822,0.3743801873$ $\mathrm{H}, 0,3.6343638388,0.7122401153,-0.2288958426$ $\mathrm{H}, 0,2.9716718981,0.8340914506,1.3920300369$ C, $0,-2.3890057551,-1.1300649866,-0.5660661122$ $\mathrm{H}, 0$, - $1.8996920797,-2.0732905232,-0.2975502174$ $\mathrm{H}, 0,-2.4549807674,-1.0796126731,-1.6592440991$ $\mathrm{H}, 0,-3.4160099495,-1.1669723532,-0.1714321428$ $\mathrm{H}, 0,0.8428278713,-2.2115477768,-0.4920807305$ $\mathrm{H}, 0,3.1118289618,-1.7457487362,0.687754732$

Sum of electronic and zero-point Energies= Sum of electronic and thermal Energies= Sum of electronic and thermal Enthalpies= Sum of electronic and thermal Free Energies=

Cyclopentylidene (10), NImag $=0, C 2$

C, $0,-0.4320472044,-1.2383611201,-0.5525321562$ C, $0,0.956647437,-0.797070389,-0.2313828039$ $\mathrm{H}, 0,1.2792643328,-0.5742167116,-1.2741734771$ $\mathrm{H}, 0,1.6316840696,-1.5923341097,0.105166026$ C, $0,0.9529565337,0.5129831274,0.596556885$ $\mathrm{H}, 0,1.8343219219,1.1401865508,0.4273432188$ $\mathrm{H}, 0,0.9230216695,0.2720852341,1.667480875$ C, $0,-1.2559523166,-0.0608162545,-0.1513891914$ $\mathrm{H}, 0,-1.6471749091,-0.4803119344,0.8036637226$ $\mathrm{H}, 0,-2.1473744109,0.1142297216,-0.7646668706$ C, $0,-0.3656876249,1.1702842941,0.1544836337$ $\mathrm{H}, 0,-0.2161025413,1.754131241,-0.7634232946$ $\mathrm{H}, 0,-0.7931410813,1.8441120603,0.9041915973$

Sum of electronic and zero-point Energies= Sum of electronic and thermal Energies= Sum of electronic and thermal Enthalpies= Sum of electronic and thermal Free Energies=
$-352.230504$

$-352.219363$

$-352.218419$

$-352.267847$

Insertion of 10 into acetonitrile, NImag=1, $-1073.8 \mathrm{~cm}^{-1}$

$\mathrm{H}, 0,1.8457873108,-1.6809385702,0.9716501167$

C, $0,2.1022810602,-0.7332687284,0.4936608507$

$\mathrm{H}, 0,3.1108387781,-0.7637686208,0.0716537132$

C, $0,0.1666902375,-0.4327288164,-1.1768977687$

$\mathrm{H}, 0,1.3877617762,-0.6496233837,-0.6220130854$

C, $0,-0.4111291896,0.9346590044,-0.9641446611$

$\mathrm{H}, 0,-0.1784012848,1.6194166089,-1.7903220855$

C, $0,-0.9021341536,-1.3890902726,-0.7326604549$

-195.106070
-195.101215
-195.100271
-195.132745 
$\mathrm{H}, \mathrm{O},-1.3338848809,-1.7186102227,-1.6989986205$

$\mathrm{C}, 0,1.8846905159,0.4018087945,1.3348509926$

$\mathrm{N}, 0,1.6176209987,1.3648699485,1.9384117355$

C, $0,-1.9740419893,-0.5978205069,0.0514646524$

$\mathrm{H}, 0,-1.6781141714,-0.516307052,1.104969948$

$\mathrm{H}, 0,-2.9657967847,-1.0603321332,0.0183599546$

C, $0,-1.916428731,0.7812234512,-0.6277272342$

$\mathrm{H}, 0,-2.5115506241,0.7747669745,-1.55024795$

$\mathrm{H}, \mathrm{O},-2.2939924973,1.5922896388,0.0018720635$

$\mathrm{H}, 0,-0.5231993885,-2.3001372099,-0.2579141647$

$\mathrm{H}, 0,0.117638275,1.3604567765,-0.0891702996$

Sum of electronic and zero-point Energies=

Sum of electronic and thermal Energies=

Sum of electronic and thermal Enthalpies=

Sum of electronic and thermal Free Energies=

$-327.810392$

$-327.801777$

$-327.800833$

$-327.844517$

Insertion of 10 into methane, NImag=1, $-877.1 \mathrm{~cm}^{-1}$

C, $0,2.3061482043,0.0020895384,0.3739912811$

$\mathrm{C}, 0,0.4483913494,0.1146339684,-1.0244904758$

$\mathrm{H}, 0,1.6976011675,0.1109527078,-0.7674424295$

C, $0,-0.3316957439,1.2496771457,-0.3726365169$

$\mathrm{H}, 0,-0.7814185918,1.8480421487,-1.1787615474$

C, $0,-0.2705259288,-1.1454108286,-0.5976044352$

$\mathrm{H}, 0,-0.9932231438,-1.3245182409,-1.4140769435$

C, $0,-1.0474472135,-0.846200203,0.7050415681$

$\mathrm{H}, 0,-0.3906192609,-0.9963619553,1.5710768105$

$\mathrm{H}, 0,-1.9201214029,-1.4933546943,0.8498782238$

C, $0,-1.4271596715,0.6435410519,0.5602227228$

$\mathrm{H}, \mathrm{O},-2.413200952,0.7342156882,0.0883106984$

$\mathrm{H}, 0,-1.4916258244,1.155748078,1.5261975924$

$\mathrm{H}, 0,0.3693492284,-2.0330746359,-0.5616638512$

$\mathrm{H}, 0,0.3381490389,1.9396089782,0.1519158068$

$\mathrm{H}, 0,2.8742237764,0.9351209547,0.3971822032$

$\mathrm{H}, 0,2.9423645822,-0.8687959195,0.2002254119$

$\mathrm{H}, 0,1.7022554064,-0.1175671468,1.2700131596$

Sum of electronic and zero-point Energies=

Sum of electronic and thermal Energies=

Sum of electronic and thermal Enthalpies=

Sum of electronic and thermal Free Energies=

$-235.560464$

$-235.553226$

$-235.552282$

$-235.591907$

Insertion of 10 into isobutane, NImag=1, $-794.7 \mathrm{~cm}^{-1}$ $\mathrm{C}, 0,1.4683160155,-0.1810202926,0.6856292169$

C, $0,-0.1173600498,0.26096052,-1.196758745$

$\mathrm{H}, 0,0.9175592767,0.0872347109,-0.4919200695$

C, $0,-0.9904997619,1.4155024001,-0.71485961$

$\mathrm{H}, 0,-0.8578644329,2.3015889716,-1.3510401495$

C, $0,-1.0367017773,-0.947008233,-1.2017369508$

$\mathrm{H}, \mathrm{0},-1.2881462999,-1.1031018184,-2.2662754644$

C, $0,-2.328490347,-0.5941300451,-0.4272170131$

$\mathrm{H}, 0,-2.1959272223,-0.792608964,0.6434882717$

$\mathrm{H}, \mathrm{0},-3.20527509,-1.1622808695,-0.7586746138$

C, $0,-2.4600462024,0.9177842012,-0.6759384698$

$\mathrm{H}, 0,-2.9368375325,1.0940384571,-1.6491383252$

$\mathrm{H}, 0,-3.067373622,1.431250124,0.0775471463$

$\mathrm{H}, 0,-0.5449171255,-1.8744149914,-0.8858091042$

$\mathrm{H}, 0,-0.6693152006,1.7321558636,0.2886790866$

C, $0,2.342196391,-1.3466192418,0.2490206055$

$\mathrm{H}, 0,2.9205024677,-1.0970999128,-0.6481827689$

$\mathrm{H}, \mathrm{O}, 1.7423935682,-2.2377832559,0.0287582667$ 
$\mathrm{H}, 0,3.0545434818,-1.6185574512,1.0426670047$

C, $0,2.2361557734,1.1140421716,0.8941027326$

$\mathrm{H}, 0,2.8351741181,1.3701889274,0.0125827527$

$\mathrm{H}, 0,2.9197287527,1.0271234693,1.7520865568$

$\mathrm{H}, 0,1.5606028531,1.9532617497,1.1010446622$

C, $0,0.5369509234,-0.5196316742,1.8320838526$

$\mathrm{H}, \mathrm{O}, 1.1175753983,-0.714472849,2.7486783615$

$\mathrm{H}, 0,-0.054667489,-1.418883378,1.6282319763$

$\mathrm{H}, \mathrm{O},-0.1508816914,0.3030823798,2.0613226962$

Sum of electronic and zero-point Energies=

Sum of electronic and thermal Energies=

Sum of electronic and thermal Enthalpies=

Sum of electronic and thermal Free Energies=

$-353.420881$

$-353.409582$

$-353.408638$

$-353.457398$

Bicyclo [4.4.0] dec-1(6) -en-2-ylidene (11), NImag=0, C2h

$\mathrm{H}, 0,1.2409371351,0.5431655258,-2.3179485412$

C, $0,1.4225981628,0.549507451,-1.2329121151$

C, $0,1.6207873062,1.9001712207,0.8200315758$

$C, 0,-0.3617951483,0.3932422826,0.5677661021$

C, $0,0.5406968111,1.0531187155,1.4092287563$

C, $0,0.2654385933,-0.139012282,-0.5595330779$

C, $0,1.6127559223,1.9729463579,-0.7154791075$

$\mathrm{H}, 0,2.5888846708,1.5316202904,1.1970429637$

$\mathrm{H}, 0,0.7655141436,2.5892230018,-1.042149222$

$\mathrm{H}, 0,2.3467395558,-0.0377163457,-1.0909331515$

$\mathrm{H}, \mathrm{O}, 1.5199858762,2.8973630823,1.2772495303$

$\mathrm{H}, 0,2.5253495722,2.4256040099,-1.1204537208$

C, $0,-0.0724965536,-1.5334505868,-1.0393189224$

$\mathrm{H}, 0,0.6710134115,-2.2322756811,-0.617566837$

$\mathrm{H}, 0,0.0679988245,-1.5876716025,-2.1277554875$

C, $0,-1.4879005242,-1.9957534641,-0.655937201$

$\mathrm{H}, \mathrm{O},-1.5880153143,-3.0714475595,-0.8469716565$

$\mathrm{H}, 0,-2.218838538,-1.4917445202,-1.3035812784$

C, $0,-1.8079447077,-1.6700388935,0.8089133433$

$\mathrm{H}, 0,-1.1065087993,-2.2039961203,1.4654532996$

$\mathrm{H}, 0,-2.8148999881,-2.0217483692,1.0658723631$

$\mathrm{C}, 0,-1.6843435,-0.1595568924,1.0628317375$

$\mathrm{H}, 0,-1.7818748045,0.0736759939,2.1279572186$

$\mathrm{H}, \mathrm{O},-2.5030639176,0.3589048415,0.5402379736$

Sum of electronic and zero-point Energies=

Sum of electronic and thermal Energies=

Sum of electronic and thermal Enthalpies=

Sum of electronic and thermal Free Energies=

$-389.167650$

$-389.158807$

$-389.157863$

$-389.201217$

Bicyclo [4.4.0] dec-1 (6)-en-2-ylidene (11), NImag=0, D2

$\mathrm{H}, 0,0.8505037169,0.7306001473,-2.3542815111$

C, $0,1.1982682378,0.588605488,-1.3188537819$

C, $0,1.6967461748,1.752698433,0.8530907539$

C, $0,-0.2984604463,0.1804054006,0.6819045318$

C, $0,0.3709902818,1.2910592568,1.4750546873$

C, $0,0.195246774,-0.2357079903,-0.5472166892$

C, $0,1.5283224741,1.935263555,-0.6591075898$

$\mathrm{H}, 0,2.4836410274,1.0079856306,1.0412159135$

$\mathrm{H}, 0,-0.3299803655,2.1315452549,1.5516532608$

$\mathrm{H}, 0,0.713424426,2.6475174158,-0.848049317$

$\mathrm{H}, 0,2.118351688,-0.0136409158,-1.4078620518$

$\mathrm{H}, 0,2.0280783693,2.6849347149,1.3257468743$

$\mathrm{H}, 0,0.5323582918,0.94354301,2.5052055214$

$\mathrm{H}, \mathrm{0}, 2.4316080101,2.3596608645,-1.1131652926$ 
$C, 0,-0.3132585967,-1.4807463875,-1.2263659632$

$\mathrm{H}, 0,0.5450750285,-2.0145886873,-1.6599172495$

C, $0,-1.6463145132,-0.1597110571,0.9172807812$

C, $0,-1.0868226352,-2.3722201089,-0.2571632723$

$\mathrm{H}, \mathrm{O},-0.3903993738,-2.7782087049,0.4877582155$

$\mathrm{H}, \mathrm{O},-1.5427402663,-3.2222678001,-0.7780978497$

C, $0,-2.1322077588,-1.4925565659,0.4482718044$

$\mathrm{H}, 0,-3.0088249276,-1.3201408169,-0.1984975774$

$\mathrm{H}, 0,-2.5579069299,-1.9929443275,1.3331887376$

$\mathrm{H}, 0,-0.9482486484,-1.2065359286,-2.0862692484$

Sum of electronic and zero-point Energies=

Sum of electronic and thermal Energies=

Sum of electronic and thermal Enthalpies=

Sum of electronic and thermal Free Energies=

$-389.168076$

$-389.159181$

$-389.158237$

$-389.201680$

Bicyclo [4.4.0] dec-1 (6) -en-2-ylidene (11), NImag=0, triplet $\mathrm{H}, 0,1.0713913718,0.6663871678,-2.3622948082$

C, $0,1.2999817101,0.5411145815,-1.2920796239$

C, $0,1.6410419208,1.8099935203,0.8644296244$

C, $0,-0.3491659947,0.2424512388,0.6353211846$

C, $0,0.2394152189,1.4435213744,1.3677586481$

C, $0,0.1544967604,-0.161905512,-0.6096623971$

C, $0,1.651986048,1.8989820517,-0.6656196387$

$\mathrm{H}, 0,2.3636109213,1.0484730504,1.1909041914$

$\mathrm{H}, 0,-0.4294061248,2.3062431299,1.2320868138$

$\mathrm{H}, 0,0.9172536748,2.651671426,-0.9848003332$

$\mathrm{H}, 0,2.1889167634,-0.114818499,-1.2679877394$

$\mathrm{H}, 0,1.9599018106,2.7604764051,1.3098446556$

$\mathrm{H}, 0,0.255195225,1.2400750155,2.4448740277$

$\mathrm{H}, 0,2.6289731647,2.2372828698,-1.0320218525$

C, $0,-0.4565117435,-1.3540610817,-1.312308745$

$\mathrm{H}, 0,0.3133060344,-1.8696969561,-1.9042310368$

C, $0,-1.4042462919,-0.4617552252,1.1762728337$

C, $0,-1.1198972091,-2.355730408,-0.3509272052$

$\mathrm{H}, 0,-0.3409796851,-2.8499024507,0.2426668767$

$\mathrm{H}, \mathrm{0},-1.6470161757,-3.1372997874,-0.9120234732$

C, $0,-2.098041146,-1.6443179848,0.6201708636$

$\mathrm{H}, 0,-3.013262917,-1.345815243,0.0821109776$

$\mathrm{H}, 0,-2.4197916173,-2.3391006013,1.4080610056$

$\mathrm{H}, \mathrm{O},-1.2024480832,-1.0037308577,-2.0473225719$

Sum of electronic and zero-point Energies=

Sum of electronic and thermal Energies=

Sum of electronic and thermal Enthalpies=

Sum of electronic and thermal Free Energies=

$-389.172350$

$-389.163524$

$-389.162580$

$-389.206908$

TS(11 $\rightarrow$ Bicyclo[4.4.0] deca-1(6),2-diene), NImag=1, - $1161.0 \mathrm{~cm}^{-1}$

$\mathrm{H}, 0,-2.0510999089,-0.5506667367,-1.6530866918$

C, $0,-1.8130596856,0.0080450118,-0.737928206$

C, $0,-1.8700015129,-0.0563260622,1.7762997873$

C, $0,0.3526113552,-0.0187663688,0.5352226969$

C, $0,-0.3522116236,-0.2740569627,1.8489427399$

C, $0,-0.3061770237,0.1588672219,-0.6366033455$

C, $0,-2.4357046738,-0.6674275239,0.48945194$

$\mathrm{H}, \mathrm{O},-2.084717316,1.0214532322,1.7872547088$

$\mathrm{H}, 0,-0.1360236818,-1.3078798387,2.1655487616$

$\mathrm{H}, 0,-2.2115094577,-1.7437811567,0.4733365093$

$\mathrm{H}, 0,-2.2553156083,1.0044379622,-0.8900718311$

$\mathrm{H}, 0,-2.3530165776,-0.4832497808,2.6637402777$

$\mathrm{H}, \mathrm{0}, 0.0913287773,0.3620020489,2.6306390743$ 
$\mathrm{H}, \mathrm{O},-3.5282033313,-0.5732556625,0.4614957896$

C, $0,0.3652247366,0.5992373731,-1.8834217951$

C, $0,1.8602254966,0.0990291256,0.5964265315$

$\mathrm{H}, 0,2.132121393,1.1231098166,0.9080060755$

$\mathrm{H}, 0,2.2549015199,-0.5601177953,1.3805492562$

C, $0,1.7663127385,0.4756817668,-1.8663038835$

$\mathrm{H}, 0,1.2236540585,1.6199015655,-1.7084023516$

$\mathrm{H}, 0,2.3259362443,0.7303883596,-2.7711620841$

C, $0,2.5230438659,-0.2219279386,-0.7491363461$

$\mathrm{H}, 0,3.5850300683,0.0476162368,-0.7439681133$

$\mathrm{H}, 0,2.4653317812,-1.3040921085,-0.941580098$

Sum of electronic and zero-point Energies= Sum of electronic and thermal Energies= Sum of electronic and thermal Enthalpies= Sum of electronic and thermal Free Energies=

$-389.147423$

$-389.138861$

$-389.137917$

$-389.180627$

Insertion of 11 into acetonitrile, NImag=1, $-1108.3 \mathrm{~cm}^{-1}$

C, $0,2.2897279745,1.1750222124,-1.6073089957$ $\mathrm{H}, 0,2.4565057021,1.622148607,-0.6254455759$ C, $0,0.1075725695,-0.1776787772,-1.0907971673$ $\mathrm{H}, 0,1.0820913878,0.6389843091,-1.6453078415$ C, $0,3.2657949653,0.1768525973,-1.9298740397$ $\mathrm{N}, 0,3.9959378869,-0.6973338862,-2.1844762324$ $\mathrm{H}, 0,2.2021023202,1.9470925339,-2.3769327402$ C, $0,0.50146002,-1.6235550286,-1.1256872034$ $\mathrm{H}, 0,1.5006791079,-1.7139329124,-1.5723411691$ $\mathrm{H}, \mathrm{O},-0.1671702753,-2.1037529223,-1.8627692541$ C, $0,-0.5086030099,0.3445385646,0.078670743$ C, $0,-1.1868058138,-0.5317696132,0.9055149401$ C, $0,-0.9219615837,-2.0138866645,0.8759465039$ $\mathrm{H}, \mathrm{O},-1.7441650976,-2.5134546183,0.3344067573$ $\mathrm{H}, \mathrm{O},-0.9754344776,-2.4032042028,1.9026307724$ C, $0,0.4180905649,-2.3471909122,0.2231356511$ $\mathrm{H}, 0,0.5357560134,-3.4302838424,0.1063244223$ $\mathrm{H}, 0,1.2337908162,-1.9994785541,0.8698903197$ C, $0,-0.6931747526,1.8514816927,0.20784118$ $\mathrm{H}, 0,0.2904060336,2.3381944691,0.1823260913$ $\mathrm{H}, 0$, - $1.227206314,2.2160247772,-0.6799863694$ C, $0,-1.4497064995,2.2637015232,1.476659154$ $\mathrm{H}, \mathrm{O},-0.799438423,2.1486651571,2.3556853883$ $\mathrm{H}, 0,-1.7195615647,3.3247757363,1.4219197501$ C, $0,-2.6949742158,1.389930754,1.6519720177$ $\mathrm{H}, \mathrm{O},-3.3335879558,1.4858504054,0.7632006012$ $\mathrm{H}, \mathrm{O},-3.2943696405,1.7178337784,2.5092076788$ C, $0,-2.2835959533,-0.0763110667,1.835311648$ $\mathrm{H}, 0,-1.9240057132,-0.2343944626,2.866302203$ $\mathrm{H}, \mathrm{O},-3.1509027222,-0.7465427463,1.7339160006$

Sum of electronic and zero-point Energies= Sum of electronic and thermal Energies= Sum of electronic and thermal Enthalpies= Sum of electronic and thermal Free Energies=

$-521.876116$

$-521.863195$

$-521.862251$

$-521.916708$

Insertion of 11 into methane, NImag=1, $-999.2 \mathrm{~cm}^{-1}$

$\mathrm{H}, 0,-2.3877192635,-0.4164849568,-0.7805133604$ C, $0,-3.1504484931,-0.6304311807,0.274566177$

C, $0,-1.5456775097,1.7523212278,-0.7300770074$ $\mathrm{H}, 0,-2.6200923482,1.9672131582,-0.7067186393$ $\mathrm{H}, \mathrm{0},-1.1769640157,2.2359747362,-1.6516854893$ C, $0,-0.8138868769,2.3570227388,0.4749528959$ 
$\mathrm{H}, \mathrm{O},-1.2498695775,1.9633045291,1.4028929553$ $\mathrm{H}, 0,-0.9193361252,3.4485690583,0.5054148689$ $\mathrm{C}, 0,0.661913652,1.960499246,0.3985779045$ $\mathrm{H}, 0,1.1916721938,2.2461378772,1.3194511341$ $\mathrm{H}, 0,1.1593450292,2.5270096449,-0.4076880095$ C, $0,0.0090465338,-1.8258392283,-0.4081000357$ $\mathrm{H}, 0,-0.8913440496,-2.3192431915,-0.0189769379$ $\mathrm{H}, 0,0.0565541679,-2.0978228022,-1.4718374975$ C, $0,2.4674892442,-1.4866806133,-0.032625612$ $\mathrm{H}, 0,3.3848355931,-1.8893772733,0.4137425837$ $\mathrm{H}, 0,2.6134848852,-1.4971739672,-1.1217394701$ C, $0,1.253228243,-2.3507263258,0.3187046175$ $\mathrm{H}, 0,1.0915597226,-2.3231955708,1.4060253892$ $\mathrm{H}, 0,1.4284564493,-3.4003420337,0.0527881888$ C, $0,-1.3140186311,0.2747068167,-0.9279818773$ C, $0,-0.1614359431,-0.3165522902,-0.2994701633$ C, $0,0.8527320297,0.4807302195,0.1648513177$ C, $0,2.241767126,-0.0457662374,0.4422621681$ $\mathrm{H}, 0,2.9873190418,0.630234704,-0.0050954167$ $\mathrm{H}, 0,2.4193007954,0.0170679186,1.5294870828$ $\mathrm{H}, 0,-4.0652158187,-0.0961773055,0.0091233383$ $\mathrm{H}, \mathrm{0},-3.2802333345,-1.7138925266,0.2434882473$ $\mathrm{H}, \mathrm{0},-2.7460095935,-0.2975082379,1.2278787225$

Sum of electronic and zero-point Energies= Sum of electronic and thermal Energies= Sum of electronic and thermal Enthalpies= Sum of electronic and thermal Free Energies=

$-429.625221$ $-429.613834$ $-429.612890$ $-429.662581$

Insertion of 11 into isobutane, NImag=1, $-970.6 \mathrm{~cm}^{-1}$ $\mathrm{H}, 0,-1.5587137368,-0.1633436078,-0.7368987652$ C, $0,-2.5584696001,-0.5258611994,0.0952065303$ C, $0,-3.6376309428,0.5145141344,-0.152382186$ $\mathrm{H}, 0,-3.766755512,0.7144607505,-1.2234322251$ $\mathrm{H}, 0,-3.4214458096,1.4623980018,0.3520178233$ $\mathrm{H}, 0,-4.6067595823,0.1579394569,0.2316110899$ C, $0,-2.8942615602,-1.8620597451,-0.556172166$ $\mathrm{H}, 0,-3.0385119317,-1.7527418201,-1.6377514924$ $\mathrm{H}, 0,-3.8274572765,-2.2685680172,-0.1354605941$ $\mathrm{H}, \mathrm{O},-2.1095321086,-2.6084379247,-0.3956945838$ C, $0,-2.1512447763,-0.6398909483,1.551823013$ H, $0,-1.2999959832,-1.3149978573,1.6905178031$ $\mathrm{H}, 0,-2.9849011995,-1.0404861135,2.1525399954$ $\mathrm{H}, \mathrm{O},-1.883085109,0.3338387441,1.9763965205$ C, $0,-0.6471488595,1.9738449955,-0.8016859399$ $\mathrm{H}, 0,-1.7095651908,2.2373557013,-0.8034343118$ $\mathrm{H}, 0,-0.2506070159,2.4231731685,-1.7298726226$ C, $0,0.0895915642,2.5871298891,0.3968490561$ $\mathrm{H}, 0,-0.3569242768,2.2193419574,1.3304000912$ $\mathrm{H}, 0,0.0058246248,3.6812084221,0.4075865857$ C, $0,1.5550374531,2.1605091767,0.3303266562$ $\mathrm{H}, 0,2.0908502884,2.4405678694,1.2496608125$ $\mathrm{H}, 0,2.0651914281,2.7120584051,-0.4791490109$ C, $0,0.8586977286,-1.6206175005,-0.45955597$ $\mathrm{H}, 0,-0.0286094934,-2.1201253168,-0.0532368226$ $\mathrm{H}, 0,0.8827333654,-1.8899484438,-1.5247367022$ C, $0,3.3262872805,-1.2926025227,-0.1447355559$ $\mathrm{H}, 0,4.2535860669,-1.698145343,0.2780589399$ $\mathrm{H}, 0,3.4445713582,-1.3032463638,-1.2372167454$ C, $0,2.1176282949,-2.150434103,0.2371741372$ $\mathrm{H}, 0,1.9836597256,-2.1207721858,1.3283786037$ $\mathrm{H}, 0,2.2806884586,-3.2013438456,-0.0317959916$ 
$C, 0,-0.4677298067,0.483555275,-0.9691543426$

C, $0,0.6847492803,-0.1097653164,-0.3469633348$

C, $0,1.7202530689,0.679185826,0.0988514956$

C, $0,3.1149086001,0.147254608,0.3371016389$

$\mathrm{H}, 0,3.8498484914,0.8232539067,-0.1280353485$

$\mathrm{H}, 0,3.321904068,0.2079850399,1.4194487581$

Sum of electronic and zero-point Energies=

Sum of electronic and thermal Energies=

Sum of electronic and thermal Enthalpies=

Sum of electronic and thermal Free Energies=

$-547.484425$

$-547.469009$

$-547.468065$

$-547.526401$

Bicyclo[2.2.1] hept-2-en-7-ylidene (12), NImag=0, (Norbornen-7-ylidene) C, $0,-0.1982337513,0.2038745041,-1.3104311721$ C, $0,1.0191718772,-0.3444854363,-0.5034153835$ $\mathrm{H}, 0,1.8979273411,-0.6328979402,-1.0805341545$ C, $0,0.9850849779,1.0926705519,-0.1303395315$ $\mathrm{H}, 0,1.7734554231,1.8124899829,-0.3351221105$ C, $0,-1.1623474229,0.2295286975,-0.0841048444$ $\mathrm{H}, 0,-2.2101724679,0.4480493396,-0.2909149449$ C, $0,-0.3214432367,1.4364519071,0.1207886845$ $\mathrm{H}, \mathrm{O},-0.6871863219,2.459948449,0.1378386451$ C, $0,-0.8951236446,-0.9494631005,0.8723193568$ $\mathrm{H}, 0,-1.579685954,-1.7713398765,0.6347671161$ $\mathrm{H}, 0,-1.0680182478,-0.6761325453,1.9192209994$ C, $0,0.57473501,-1.3362209176,0.5897973435$ $\mathrm{H}, 0,1.2082918642,-1.2750885926,1.4816906873$ $\mathrm{H}, 0,0.6543255057,-2.3591660547,0.2053670418$

Sum of electronic and zero-point Energies= Sum of electronic and thermal Energies= Sum of electronic and thermal Enthalpies= Sum of electronic and thermal Free Energies=

$-271.274905$

$-271.269416$

$-271.268472$

$-271.303773$

Insertion of 12 into acetonitrile, NImag=1, $-1092.0 \mathrm{~cm}^{-1}$

C, $0,-2.1643197371,0.7965302527,0.7364959614$ $\mathrm{H}, 0,-2.011959093,1.7473608655,0.2246356655$ C, $0,0.234565984,0.1658020272,1.0204915437$ $\mathrm{H}, \mathrm{O},-0.9249050258,0.4128846105,1.373934734$ $\mathrm{C}, 0,-2.6970225726,-0.2275199972,-0.1031641342$ $\mathrm{N}, 0,-3.062980123,-1.0986310301,-0.7893282698$ $\mathrm{H}, 0,-2.7238763478,0.9289856272,1.6663490144$ C, $0,0.5938883208,-1.0331564522,0.1529004222$ $\mathrm{H}, 0,0.0791141185,-1.9717347908,0.3558486804$ C, $0,1.1231352598,1.1726512025,0.3057392722$ $\mathrm{H}, 0,1.091395477,2.2071827324,0.6505952997$ C, $0,0.9060411806,0.9590902198,-1.2243636703$ $\mathrm{H}, 0,1.7950328063,1.2363560114,-1.8003221128$ $\mathrm{H}, 0,0.0792206688,1.5869493187,-1.5738607506$ C, $0,0.5561472428,-0.5503276348,-1.330059162$ $\mathrm{H}, 0,1.2662906507,-1.1084845435,-1.9483144456$ $\mathrm{H}, \mathrm{O},-0.4446174589,-0.7084782762,-1.7406967412$ C, $0,2.0136283873,-0.9370091179,0.6867265964$ C, $0,2.3304562372,0.3761293788,0.7750194306$ $\mathrm{H}, 0,2.617096483,-1.7786055381,1.0121051246$ $\mathrm{H}, 0,3.2389467656,0.80486192,1.1863058599$

Sum of electronic and zero-point Energies= Sum of electronic and thermal Energies= Sum of electronic and thermal Enthalpies=

$-403.965223$

$-403.955834$

$-403.954890$ 
Insertion of 12 into methane, NImag=1, $-1131.2 \mathrm{~cm}^{-1}$ $\mathrm{H}, 0,0.0175900487,-0.4862021242,2.1561704301$ C, $0,-0.2439412091,-0.2199800485,1.1301870553$ C, $0,-1.6315285473,-0.6523413964,-0.6728035815$ C, $0,-0.2439412091,-0.2199800485,-1.1301870553$ C, $0,-1.6315285473,-0.6523413964,0.6728035815$ $\mathrm{H}, 0,0.0175900487,-0.4862021242,-2.1561704301$ $\mathrm{H}, 0,-2.4517410442,-0.9122174123,1.3347412611$ $\mathrm{H}, 0$, - 2.4517410442, - $0.9122174123,-1.3347412611$ C, $0,0.4394228812,-0.9953530992,0$. C, $0,2.6264189535,-0.306586743,0$. $\mathrm{H}, 0,2.6520597304,0.2939365155,0.9071402586$ $\mathrm{H}, 0,2.6520597304,0.2939365155,-0.9071402586$ $\mathrm{H}, 0,3.418031969,-1.0623186344,0$. $\mathrm{H}, 0,1.6230237069,-1.250396593,0$. C, $0,-0.1002793483,1.3007284231,0.7774729642$ $\mathrm{H}, 0,0.8362531782,1.7025309749,1.178589768$ $\mathrm{H}, 0,-0.9177206271,1.8936873167,1.2016329734$ C, $0,-0.1002793483,1.3007284231,-0.7774729642$ $\mathrm{H}, \mathrm{O}, 0.8362531782,1.7025309749,-1.178589768$ $\mathrm{H}, 0,-0.9177206271,1.8936873167,-1.2016329734$

Sum of electronic and zero-point Energies= Sum of electronic and thermal Energies= Sum of electronic and thermal Enthalpies= Sum of electronic and thermal Free Energies=
$-311.714386$

$-311.706735$

$-311.705791$

$-311.746169$

Insertion of 12 into isobutane, NImag=1, $-975.6 \mathrm{~cm}^{-1}$ C, $0,-1.9816561265,0.0467998489,-0.036740281$ $\mathrm{C}, 0,0.3853007529,0.1183666796,-0.8567310916$ $\mathrm{H}, 0,-0.7763389557,0.1777064224,-0.9010616317$ C, $0,-2.7791465894,0.8182016476,-1.0780138924$ $\mathrm{H}, \mathrm{O},-2.498235428,1.8781562379,-1.0973016183$ $\mathrm{H}, 0,-3.857745442,0.7659715809,-0.8568532694$ $\mathrm{H}, \mathrm{O},-2.6307945978,0.4125606902,-2.0866675291$ C, $0,-2.2579696712,-1.4414587793,-0.0518811908$ $\mathrm{H}, 0,-3.3172288805,-1.6488528026,0.1768570836$ $\mathrm{H}, 0,-1.6552426784,-1.9775447898,0.6897704095$ $\mathrm{H}, \mathrm{O},-2.0521419169,-1.8760607268,-1.0384845406$ C, $0,1.1557630334,-1.1028734,-0.3581859422$ $\mathrm{H}, 0,0.7990272439,-2.0887707615,-0.66156556$ C, $0,1.2715566249,1.1417714793,-0.1473765934$ $\mathrm{H}, 0,1.0183915055,2.1982621808,-0.2564388686$ C, $0,1.3143011368,-0.9020315275,1.1891535028$ $\mathrm{H}, 0,2.1868193855,-1.43314848,1.5854982595$ $\mathrm{H}, 0,0.4314234501,-1.2881406345,1.7110840652$ C, $0,1.4274782574,0.6414862585,1.3324144545$ $\mathrm{H}, \mathrm{O}, 2.3837962767,0.9566153835,1.7638359532$ $\mathrm{H}, 0,0.6336538789,1.0484390759,1.9649650063$ C, $0,2.4617400839,-0.6510566406,-1.0038058371$ $\mathrm{H}, 0,3.1901053937,-1.3119953803,-1.4635205096$ C, $0,2.5274432548,0.68763607,-0.8855669982$ $\mathrm{H}, 0,3.3208452975,1.3455380488,-1.2268312586$ C, $0,-2.0257499571,0.6875517415,1.3317738855$ $\mathrm{H}, \mathrm{O},-1.4479360935,0.1263887019,2.0729033304$ $\mathrm{H}, \mathrm{O},-3.0663722063,0.7287715424,1.7000637048$ $\mathrm{H}, 0,-1.6563910325,1.7197434421,1.3135068771$ 
Sum of electronic and thermal Energies=

$-429.559141$

Sum of electronic and thermal Enthalpies=

$-429.558197$

Sum of electronic and thermal Free Energies=

$-429.608270$

Bicyclo[2.1.1] hex-2-en-5-ylidene (13), NImag=0, C, $0,-0.0764861976,-1.1843793048,-0.1279365324$ C, $0,1.0828637595,-0.0260609196,-0.2371669218$ C, $0,0.56075732,-0.0924762793,1.1218741947$ C, $0,-0.8196973143,0.0148421536,0.9536657519$ C, $0,-0.9858993457,0.1347675559,-0.4892457745$ $\mathrm{H}, 0,2.1242758469,-0.263101614,-0.4341917025$ $\mathrm{H}, 0,1.0705622508,-0.4849429061,1.9928227803$ $\mathrm{H}, 0,-1.5763171868,-0.2791708756,1.6703004334$ $\mathrm{H}, 0,-1.9737584072,0.0554851783,-0.9335373118$ C, $0,0.2008939408,0.8812359673,-1.0864621629$ $\mathrm{H}, 0,0.2701808428,1.9736467608,-0.958119084$ $\mathrm{H}, 0,0.3104636778,0.6305084181,-2.145646446$

Sum of electronic and zero-point Energies= Sum of electronic and thermal Energies= Sum of electronic and thermal Enthalpies= Sum of electronic and thermal Free Energies=

\section{(Homopyramidane)}

Complex between 13 and acetonitrile, NImag=0 C, $0,2.5627341793,1.5435693743,-0.3251404691$ $\mathrm{H}, \mathrm{0}, 2.645556352,2.6205480188,-0.1461703814$ C, $0,-0.0806358028,-0.180490539,1.0255044048$ $\mathrm{H}, 0,1.8773246563,1.0947034443,0.4112993791$ C, $0,-0.5769133948,-1.627651986,0.4431566684$ $\mathrm{H}, 0,0.1143734357,-2.4164972476,0.1620429451$ C, $0,-1.6021682644,-0.2228378843,1.603731904$ $\mathrm{H}, \mathrm{O},-1.9219791993,0.3677208293,2.4571919556$ C, $0,-1.681739861,-1.7409852042,1.4885594698$ $\mathrm{H}, 0,-2.6381892111,-2.2077610792,1.2064754098$ $\mathrm{H}, 0,-1.2979077378,-2.2112913485,2.3985436612$ C, $0,-0.9009673828,-0.5688722345,-0.5055916516$ C, $0,-1.5841105787,0.3694621125,0.2689638888$ $\mathrm{H}, 0,-0.4192169041,-0.3790329928,-1.4570230593$ $\mathrm{H}, 0,-1.7394544645,1.4114264907,0.0192625826$ $\mathrm{H}, 0,3.5560428897,1.0939471874,-0.2259442347$ C, $0,2.0216804136,1.2901819639,-1.657507368$ $\mathrm{N}, 0,1.5537391903,1.0645690117,-2.6965341915$

Sum of electronic and zero-point Energies= Sum of electronic and thermal Energies= Sum of electronic and thermal Enthalpies= Sum of electronic and thermal Free Energies=

$-231.963380$ $-231.958761$ $-231.957817$ $-231.990673$

Insertion of 13 into acetonitrile, NImag=1, $-1067.7 \mathrm{~cm}^{-1}$ $\mathrm{C}, 0,-2.1163632084,0.8169411792,0.603795749$ $\mathrm{H}, \mathrm{O},-2.0554728149,1.7517854843,0.0464544617$ C, $0,0.3185905368,0.3254270975,0.8418310946$ $\mathrm{H}, 0,-0.7609927887,0.577275383,1.2861072848$ C, $0,-2.544636747,-0.2985130498,-0.1696070933$ $\mathrm{N}, 0,-2.8132895576,-1.2448281798,-0.8009484774$ $\mathrm{H}, 0,-2.6598814267,0.9368678939,1.5440595124$ C, $0,0.6995266515,-0.8765904122,-0.0526765362$ $\mathrm{H}, 0,0.1647924961,-1.8233762222,-0.0512228772$ C, $0,1.1571465931,1.126244471,-0.1830282011$

$-364.682320$

$-364.672456$

$-364.671512$

$-364.719937$ 
$\mathrm{H}, 0,1.1020885246,2.2065084792,-0.3084628812$ C, $0,0.7852011394,0.0823915718,-1.2711113884$ $\mathrm{H}, 0,1.5300333616,-0.1373177222,-2.0475946065$ $\mathrm{H}, 0,-0.1985769409,0.2625227523,-1.7120229987$ C, $0,2.0938585545,-0.7936754288,0.5614428165$ C, $0,2.3971675506,0.5256952338,0.4734508123$ $\mathrm{H}, 0,2.6099852121,-1.578620412,1.1009414316$ $\mathrm{H}, 0,3.2181048568,1.0706276471,0.9237964941$

Sum of electronic and zero-point Energies= Sum of electronic and thermal Energies= Sum of electronic and thermal Enthalpies= Sum of electronic and thermal Free Energies=

$-364.637126$

$-364.628530$

$-364.627586$

$-364.671699$

Insertion of 13 into methane, NImag=1, $-1237.6 \mathrm{~cm}^{-1}$ C, $0,-2.6275473451,-0.0715270897,-0.0294535379$ $\mathrm{C}, 0,-0.4758204468,-0.0588409961,-0.7864837281$ $\mathrm{H}, 0,-1.627598937,-0.1053545672,-1.058203718$ $\mathrm{C}, 0,0.3008401696,-1.0069650324,0.1818619224$ $\mathrm{H}, 0,0.0488830641,-2.0573062877,0.3317581683$ C, $0,0.2466102694,1.0358369535,0.0618180452$ $\mathrm{H}, 0,-0.0609897216,2.0817035072,0.0885297334$ C, $0,0.1995190083,0.0813431839,1.2939526487$ $\mathrm{H}, 0,1.0240004993,0.1457483009,2.0174882341$ $\mathrm{H}, 0,-0.7656420856,0.0858673777,1.8070692782$ C, $0,1.647567407,-0.6581388906,-0.4610402608$ $\mathrm{H}, 0,2.3719291597,-1.3566607315,-0.8626305161$ C, $0,1.6118884933,0.6858588151,-0.5400183787$ $\mathrm{H}, \mathrm{0}, 2.2995467586,1.3698943486,-1.0228530163$ $\mathrm{H}, 0,-3.4369714104,-0.1360665869,-0.7633838458$ $\mathrm{H}, 0,-2.659791042,0.8700202184,0.5159983903$ $\mathrm{H}, 0,-2.6117116191,-0.9432472409,0.6224070273$

Sum of electronic and zero-point Energies= Sum of electronic and thermal Energies= Sum of electronic and thermal Enthalpies= Sum of electronic and thermal Free Energies=

$-272.383921$ $-272.377090$ $-272.376146$ $-272.414313$

Insertion of 13 into isobutane, NImag=1, $-1048.8 \mathrm{~cm}^{-1}$ C, $0,-1.8573031464,-0.0455294864,-0.0758626157$ $\mathrm{C}, 0,0.5774908628,-0.0964677109,-0.6597105453$ $\mathrm{H}, 0,-0.5378540923,-0.1539315919,-0.8778558086$ $\mathrm{C}, 0,-2.354824333,1.1496650892,-0.8658439894$ $\mathrm{H}, 0,-1.9375619466,2.0885254762,-0.4824661816$ $\mathrm{H}, 0,-3.4537930339,1.2330015039,-0.8115551899$ $\mathrm{H}, 0,-2.0908651159,1.0736849475,-1.9291459515$ C, $0,-2.3546512722,-1.3789021237,-0.6028658237$ $\mathrm{H}, 0,-3.4531981126,-1.4493137648,-0.5290965489$ $\mathrm{H}, \mathrm{O},-1.9352097627,-2.2185462855,-0.0356745395$ $\mathrm{H}, \mathrm{O},-2.0931894952,-1.5233507123,-1.6596564252$ C, $0,1.4212411685,-0.9630361787,0.3179038284$ $\mathrm{H}, 0,1.1741619264,-1.9896776192,0.5912074027$ C, $0,1.3794478728,1.0591118759,0.0035895257$ $\mathrm{H}, 0,1.0890320826,2.1092729711,-0.0459953413$ C, $0,1.4201314812,0.2292503778,1.3231861703$ $\mathrm{H}, 0,2.3009919875,0.3480303033,1.9704608253$ $\mathrm{H}, 0,0.5001317226,0.3000911319,1.9054113926$ C, $0,2.7255875111,-0.7011317567,-0.4501292425$ $\mathrm{H}, 0,3.4182306287,-1.4469570752,-0.821684099$ C, $0,2.6982177425,0.6293556754,-0.6568937592$ $\mathrm{H}, 0,3.3627479626,1.2542839799,-1.2416296815$ 
C, $0,-2.0099167427,0.1067096665,1.4191400069$

$\mathrm{H}, 0,-1.543699268,-0.7176040292,1.9711127068$

$\mathrm{H}, 0,-3.0804798537,0.1071862984,1.695773541$

$\mathrm{H}, 0$, - 1.5919724981,1.0511518959, 1.7857125661

Sum of electronic and zero-point Energies= Sum of electronic and thermal Energies= Sum of electronic and thermal Enthalpies= Sum of electronic and thermal Free Energies=

$-390.240394$

$-390.229172$

$-390.228228$

$-390.277884$

2-Azabicyclo [4.4.0] dec-1 (6)-en-7-ylidene (14), NImag=0

C, $0,1.2015855273,0.5636771289,-1.4239650884$ C, $0,1.5242811917,2.0044652036,0.7209039449$ C, $0,-0.2032598936,0.208442475,0.5462345189$ C, $0,0.1183776147,1.5654003911,1.1254017868$ C, $0,0.4508842736,-0.283822842,-0.5969531368$ C, $0,1.6661759938,1.8576821904,-0.8051732333$ $\mathrm{H}, 0,2.2575529737,1.3561330819,1.2188548563$ $\mathrm{H}, \mathrm{0},-0.6216983971,2.2922243466,0.753333564$ $\mathrm{H}, 0,1.0970206061,2.6575324804,-1.3135093251$ $\mathrm{H}, 0,1.7142617936,3.0299218607,1.0621680364$ $\mathrm{H}, 0,0.0014323018,1.5341485308,2.2176893961$ $\mathrm{H}, 0,2.705871759,2.0402483086,-1.1144755511$ C, $0,0.0644007309,-1.6817685764,-1.0666371944$ $\mathrm{H}, 0,0.6806294342,-2.4418679394,-0.5607186011$ $\mathrm{H}, 0,0.2892825712,-1.7482534125,-2.1347558917$ C, $0,-1.4175881043,-1.9776135824,-0.7909612357$ $\mathrm{H}, 0,-1.681499405,-3.008137035,-1.0551271061$ $\mathrm{H}, \mathrm{O},-2.0437866839,-1.3121839737,-1.3986838109$ C, $0,-1.7327618759,-1.7515732114,0.6885518687$ $\mathrm{H}, 0,-1.2712971496,-2.5403103693,1.3005796057$ $\mathrm{H}, 0,-2.8120557196,-1.7811442598,0.8750345325$ $\mathrm{N}, 0,-1.2343506247,-0.4443727755,1.1238247895$ $\mathrm{H}, \mathrm{O},-1.7078324618,0.0029627495,1.8984233848$

Sum of electronic and zero-point Energies= Sum of electronic and thermal Energies= Sum of electronic and thermal Enthalpies= Sum of electronic and thermal Free Energies=

$-405.229223$

$-405.220403$

$-405.219459$

$-405.262717$

2-Azabicyclo [4.4.0] dec-1 (6) -en-7-ylidene (14), NImag=0

C, $0,1.0060607492,0.8449875011,-1.4825008306$ $\mathrm{C}, 0,1.2802728617,2.1139746657,0.7757680533$ C, $0,-0.3029480424,0.1983908975,0.495823978$ C, $0,-0.0622382977,1.5160866567,1.1917084657$ C, $0,0.3122858762,-0.1135134663,-0.7272396988$ C, $0,1.3724425748,2.1168910686,-0.7615744794$ $\mathrm{H}, 0,2.0888484564,1.4940113076,1.1856127494$ $\mathrm{H}, 0,-0.8772242415,2.2064653692,0.9199659243$ $\mathrm{H}, 0,0.7047909336,2.8953449608,-1.1759135773$ $\mathrm{H}, 0,1.3958082109,3.1185008602,1.2023715989$ $\mathrm{H}, 0,-0.1251618283,1.3740088138,2.279622483$ $\mathrm{H}, \mathrm{0}, 2.3748824705,2.4343529245,-1.0840415576$ C, $0,0.0108333465,-1.4778001409,-1.3421470269$ $\mathrm{H}, 0,0.9091803805,-1.8389852626,-1.8545164736$ C, $0,-1.6110434091,-1.909090266,0.5284803514$ $\mathrm{H}, 0,-1.8823643974,-2.5628468613,1.3651991016$ $\mathrm{N}, 0,-1.2123905595,-0.6126443199,1.0777619003$ $\mathrm{H}, 0,-1.6982357286,-0.277728163,1.9003030805$ C, $0,-0.4591158924,-2.4934966954,-0.2927203772$ $\mathrm{H}, 0,0.3662918885,-2.7408727509,0.3874479819$ 
$\mathrm{H}, 0,-0.7880077148,-3.4290748274,-0.7596879514$ $\mathrm{H}, 0,-0.7563687794,-1.3731120894,-2.1230370443$ $\mathrm{H}, 0$, - 2.5050043346, - $1.7901353683,-0.1012502312$

Sum of electronic and zero-point Energies= Sum of electronic and thermal Energies= Sum of electronic and thermal Enthalpies= Sum of electronic and thermal Free Energies=

$-405.228816$

$-405.219951$

$-405.219007$

$-405.262395$

2-Azabicyclo[4.4.0] dec-1(6)-en-7-ylidene (14), NImag=0, triplet C, $0,1.3617096921,0.4968226936,-1.2070283543$ C, $0,1.5128077992,1.9982796908,0.7315028894$ $C, 0,-0.2981539612,0.2506134399,0.4935396957$ C, $0,0.0498134674,1.6188401979,1.0253492737$ C, $0,0.3734237187,-0.2653091945,-0.628326036$ C, $0,1.8605510743,1.8217489726,-0.7712112479$ $\mathrm{H}, 0,2.1737487119,1.350128872,1.3197241347$ $\mathrm{H}, 0,-0.6236059173,2.3757425465,0.5898787343$ $\mathrm{H}, 0,1.402491818,2.6357876183,-1.3582752717$ $\mathrm{H}, 0,1.7059617437,3.031787691,1.0444824929$ $\mathrm{H}, 0,-0.1247864052,1.6561696059,2.1118038589$ $\mathrm{H}, 0,2.9461960797,1.9208071307,-0.9111719747$ $\mathrm{C}, 0,-0.0172963251,-1.6329298357,-1.1738868454$ $\mathrm{H}, 0,0.6844405294,-2.3938478497,-0.8027553781$ $\mathrm{H}, 0,0.0772706097,-1.6361274893,-2.2656661631$ C, $0,-1.4465873035,-2.0102692029,-0.7587856182$ $\mathrm{H}, 0,-1.6581872417,-3.0564439437,-1.008808236$ $\mathrm{H}, \mathrm{0},-2.1706594714,-1.3892420145,-1.3008489982$ C, $0,-1.6394837377,-1.7934272729,0.7434399362$ $\mathrm{H}, 0,-0.9551288053,-2.4627197198,1.2953514235$ $\mathrm{H}, 0,-2.6621412774,-2.0468968093,1.0459483445$ $\mathrm{N}, 0,-1.3992825108,-0.3897917556,1.0667326822$ $\mathrm{H}, 0,-1.5413293442,-0.1628202815,2.0456460985$

Sum of electronic and zero-point Energies= Sum of electronic and thermal Energies= Sum of electronic and thermal Enthalpies= Sum of electronic and thermal Free Energies=

$-405.217600$ $-405.208862$ $-405.207918$ $-405.251976$

TS (14 $\rightarrow$ 2-Azabicyclo[4.4.0] deca-1(6), 7-diene), NImag=1, $-1357.0 \mathrm{~cm}^{-1}$ $\mathrm{H}, 0,-2.1535761139,-0.4245358273,-1.6413255116$ C, $0,-1.8441789669,0.0850841916,-0.7208056733$ C, $0,-1.7928150872,-0.1111406973,1.7936242695$ C, $0,0.3166450152,-0.0202305791,0.5277868225$ $\mathrm{N}, 0,-0.3440252161,-0.2646343595,1.7112113332$ C, $0,-0.3258159405,0.1515609918,-0.6703636741$ C, $0,-2.4293761349,-0.6354381513,0.5030160628$ $\mathrm{H}, 0,-2.0708160526,0.945750995,1.9417897384$ $\mathrm{H}, 0,-2.2313937907,-1.7122867768,0.4309518225$ $\mathrm{H}, 0,-2.2635051928,1.1007400562,-0.7932320268$ $\mathrm{H}, 0,-2.1503383053,-0.6707693645,2.6656577871$ $\mathrm{H}, 0,-3.5169330092,-0.5066649452,0.5543746167$ C, $0,0.3843735684,0.4614823134,-1.9042151062$ C, $0,1.817390307,0.1309806466,0.6482502996$ $\mathrm{H}, 0,2.0366404991,1.1707328101,0.9433611928$ $\mathrm{H}, 0,2.1959306402,-0.5051613846,1.4588375851$ C, $0,1.7868711942,0.3991279035,-1.8457711475$ $\mathrm{H}, 0,1.1655175644,1.5412578047,-1.9023430411$ $\mathrm{H}, 0,2.3593153046,0.5521234268,-2.764634558$ C, $0,2.5398704963,-0.1926851265,-0.6642714751$ $\mathrm{H}, 0,3.5828393441,0.1415349498,-0.6258247704$ 
$\mathrm{H}, 0,2.5585973699,-1.2835621474,-0.8133535539$

$\mathrm{H}, 0,0.1781115448,-0.1091680369,2.5637591184$

Sum of electronic and zero-point Energies=

Sum of electronic and thermal Energies=

Sum of electronic and thermal Enthalpies=

Sum of electronic and thermal Free Energies=

$-405.197893$

$-405.189267$

$-405.188323$

$-405.231159$

TS(14 $\rightarrow$ 2-Azabicyclo[4.4.0] deca-1(6),7-diene), NImag=1, - $1322.4 \mathrm{~cm}^{-1}$ $\mathrm{H}, \mathrm{0},-2.2818870686,1.4334316766,0.3697388397$ $\mathrm{C}, 0,-1.8183928191,0.468976914,0.6163566452$ C, $0,-1.6285721628,-1.9296865166,-0.1443738361$ C, $0,0.3499274609,-0.4812739298,-0.1984246292$ $\mathrm{N}, 0,-0.2686172338,-1.6479896364,-0.5941704622$ C, $0,-0.3170761602,0.5570064598,0.3992960644$ C, $0,-2.458482912,-0.6448847304,-0.2271917743$ $\mathrm{H}, 0,-1.6337208674,-2.3072652007,0.8920024769$ $\mathrm{H}, \mathrm{O},-2.5111215846,-0.3357224624,-1.2787932347$ $\mathrm{H}, \mathrm{0},-2.0312250627,0.3082380225,1.6834816556$ $\mathrm{H}, \mathrm{O},-2.0486556668,-2.7138379878,-0.7843995348$ $\mathrm{H}, 0,-3.4837555546,-0.848472797,0.1035552022$ C, $0,0.4216962632,1.6400410636,1.0219732934$ C, $0,1.847939587,-0.4274844944,-0.4155418947$ $\mathrm{H}, 0,2.3528856701,-0.891595841,0.4465911316$ $\mathrm{H}, 0,2.1176699661,-1.0277110323,-1.2939934331$ $\mathrm{C}, 0,1.6980340823,1.8974609682,0.4819532109$ $\mathrm{H}, 0,2.3481415665,2.622580134,0.9793114033$ $\mathrm{H}, 0,0.7228371903,2.7108983544,0.2882949607$ C, $0,2.3296356623,1.0169265504,-0.5948930729$ $\mathrm{H}, 0,3.4237322169,1.0711597531,-0.5635833659$ $\mathrm{H}, 0,2.0229783616,1.3816153658,-1.5853845913$ $\mathrm{H}, 0,0.3341874599,-2.4498842391,-0.7325523156$

Sum of electronic and zero-point Energies= Sum of electronic and thermal Energies= Sum of electronic and thermal Enthalpies= Sum of electronic and thermal Free Energies=

$-405.198313$ $-405.189752$ $-405.188808$ $-405.231468$

Complex between 14 and ancetonitrile, NImag=0 C, $0,-1.0745847704,0.6977014068,-0.2532415983$ $\mathrm{N}, 0,-2.2888243182,-3.9079028504,0.0090652063$ C, $0,-1.3956710467,2.15600839,-0.0530809898$ $\mathrm{H}, \mathrm{O},-2.3968774863,2.250158902,0.3910416474$ $\mathrm{H}, 0,-1.5146521758,2.5776166108,-1.0688979644$ C, $0,0.2516456879,0.3078606343,-0.0361759194$ C, $0,1.2938717998,1.2424881711,0.1241826919$ C, $0,1.023721367,2.7253586265,0.2052678223$ $\mathrm{H}, 0,1.1198662575,3.1455198602,-0.8087792266$ $\mathrm{H}, 0,1.7955313173,3.2106435047,0.818451745$ C, $0,-0.3803176845,2.9950243593,0.7425745161$ $\mathrm{H}, \mathrm{O},-0.6037579791,4.0680009334,0.6940576536$ $\mathrm{H}, 0,-0.4207118974,2.7041566513,1.8006446202$ C, $0,0.6430050982,-1.1644859381,-0.1358899653$ $\mathrm{H}, 0,-0.1480787821,-1.7803990064,0.3017602356$ $\mathrm{H}, 0,0.7057031839,-1.4636929384,-1.1926612128$ C, $0,1.9836230125,-1.4506142277,0.551923905$ $\mathrm{H}, 0,1.885808599,-1.311444083,1.6362483919$ $\mathrm{H}, 0,2.3025444297,-2.4849960432,0.3841027221$ C, $0,3.0614642163,-0.5004320181,0.0286062101$ $\mathrm{H}, 0,3.3021405807,-0.7309545454,-1.0187463781$ $\mathrm{H}, 0,3.9889790688,-0.5812191918,0.6062029136$ 
$\mathrm{N}, 0,2.5891046522,0.8840365942,0.1289426258$

$\mathrm{H}, 0,3.2864427213,1.6168500372,0.17640271$

C, $0,-3.3997507102,-1.7107155807,-0.888025101$

$\mathrm{H}, 0,-2.6874384372,-0.8682770762,-0.7403329601$

$\mathrm{H}, 0,-3.6331341635,-1.8058052807,-1.9541785436$

$\mathrm{H}, 0,-4.3282651934,-1.4997789631,-0.3462427827$

C, $0,-2.7996840337,-2.9447464198,-0.3936629709$

Sum of electronic and zero-point Energies=

Sum of electronic and thermal Energies=

Sum of electronic and thermal Enthalpies=

Sum of electronic and thermal Free Energies=

$-537.950955$

$-537.936960$

$-537.936016$

$-537.994399$

Insertion of 14 into acetonitrile, NImag=1, $-1192.8 \mathrm{~cm}^{-1}$

C, $0,-1.0357675982,-0.1367528321,1.2895640612$

$\mathrm{N}, 0,-1.4494725645,1.229645325,-2.2903234659$

C, $0,-1.5188080596,-1.5643861464,1.2561816095$

$\mathrm{H}, 0,-2.6135560594,-1.5794968152,1.1764294856$

$\mathrm{H}, 0,-1.2963062057,-1.9975219019,2.2474319875$

$\mathrm{C}, 0,0.2121688208,0.1525401267,0.7383813617$

C, $0,1.0490340471,-0.8536695883,0.2178130298$

C, $0,0.6321781564,-2.3038654028,0.2090173769$

$\mathrm{H}, 0,1.0163311619,-2.7764367305,1.127065067$

$\mathrm{H}, 0,1.1096061332,-2.8216428175,-0.6336985609$

C, $0,-0.8911096199,-2.4395576472,0.1593864466$

$\mathrm{H}, \mathrm{0},-1.1759459838,-3.4932517123,0.2670659623$

$\mathrm{H}, 0,-1.2495419091,-2.1000727509,-0.8203021794$

C, $0,0.7297410665,1.5834532411,0.7088271369$

$\mathrm{H}, 0,-0.1170469811,2.269438658,0.6104259911$

$\mathrm{H}, 0,1.2148425405,1.8274810487,1.6658433905$

C, $0,1.7184966685,1.7942412168,-0.4439270448$

$\mathrm{H}, 0,1.1870771093,1.6848123322,-1.3961085752$

$\mathrm{H}, 0,2.1545305131,2.7989354166,-0.4158682529$

$\mathrm{C}, 0,2.8446375844,0.763200409,-0.364015315$

$\mathrm{H}, 0,3.4966001746,0.9622800005,0.4983155651$

$\mathrm{H}, 0,3.4703522736,0.7817541033,-1.2627856875$

$\mathrm{N}, 0,2.2801662434,-0.5868291616,-0.2414228529$

$\mathrm{H}, 0,2.8365355505,-1.3605434265,-0.5825612602$

C, $0,-2.810140253,1.3017469054,-0.0796678702$

$\mathrm{H}, \mathrm{O},-2.0095681198,0.7643706705,0.8904983977$

$\mathrm{H}, \mathrm{O},-3.0212313681,2.3142225491,0.2758789143$

$\mathrm{H}, 0,-3.7251607761,0.7041321944,-0.1019167927$

C, $0,-2.112493114,1.290020724,-1.3288089963$

Sum of electronic and zero-point Energies=

Sum of electronic and thermal Energies=

Sum of electronic and thermal Enthalpies=

$-537.941391$

$-537.928627$

$-537.927683$

Sum of electronic and thermal Free Energies=

$-537.981363$

Insertion of 14 into acetonitrile, NImag=1, $-1204.3 \mathrm{~cm}^{-1}$

C, $0,-1.0426956269,0.5131812676,-1.0558390231$

$\mathrm{N}, 0,-1.7434225671,-1.8899434707,1.8827971173$

C, $0,-1.5330112797,1.8355450083,-0.522302411$

$\mathrm{H}, 0,-2.6270960555,1.8098476131,-0.4375793097$

$\mathrm{H}, 0,-1.3232471362,2.589381569,-1.3017553765$

C, $0,0.1883628754,0.0326542606,-0.609844005$

C, $0,1.0416512895,0.8225712745,0.1827204345$

C, $0,0.6233821634,2.1809369928,0.6901071628$

H, $0,0.9920877214,2.9443539429,-0.0134574252$

$\mathrm{H}, 0,1.1123283949,2.3803667804,1.653188696$

C, $0,-0.8991951017,2.2758591916,0.8052187051$ 
$\mathrm{H}, \mathrm{O},-1.1912870726,3.2973424475,1.076958656$

$\mathrm{H}, 0,-1.2414077353,1.6093119773,1.6062869909$ $\mathrm{C}, 0,0.686910132,-1.3431288261,-1.0312728051$ $\mathrm{H}, 0,0.205304134,-1.6223702824,-1.97343009$ C, $0,2.8818973439,-0.8443522951,0.0859650556$ $\mathrm{H}, 0,3.9580338051,-0.6985224921,-0.0562063043$ $\mathrm{N}, 0,2.2989859372,0.4452442981,0.4671621611$ $\mathrm{H}, 0,2.8551541946,1.0559393819,1.051793759$ C, $0,-2.8683154004,-1.300554624,-0.3822375253$ $\mathrm{H}, \mathrm{O},-2.0217012644,-0.4535451644,-1.0533902655$ $\mathrm{H}, \mathrm{O},-2.9687759629,-2.1548816914,-1.0575999797$ $\mathrm{H}, 0,-3.8199571888,-0.7718180243,-0.2835757647$ C, $0,-2.299678901,-1.6601285036,0.8802289461$ C, $0,2.2140653411,-1.3649486391,-1.1870195675$ $\mathrm{H}, 0,2.5133700256,-0.7336426314,-2.0333928089$ $\mathrm{H}, 0,2.5777685913,-2.3781825918,-1.3897265751$ $\mathrm{H}, 0,0.383893409,-2.087174187,-0.2813417932$ $\mathrm{H}, 0,2.746351535,-1.5593230844,0.9091628401$

Sum of electronic and zero-point Energies= Sum of electronic and thermal Energies= Sum of electronic and thermal Enthalpies= Sum of electronic and thermal Free Energies=

$-537.940732$ $-537.927854$ $-537.926910$ $-537.981543$
Insertion of 14 into methane, NImag=1, $-1151.6 \mathrm{~cm}^{-1}$ C, $0,-1.3023478763,0.2879681334,-0.9341159872$ C, $0,-0.1434214866,-0.3185915669,-0.3866014029$ $\mathrm{H}, 0,-2.3512584114,-0.3500564242,-0.8139231118$ C, $0,-3.2244633495,-0.5990754139,0.3038842795$ C, $0,-1.4854276095,1.7738431884,-0.7373611649$ $\mathrm{H}, 0,-2.5540427532,2.0147514894,-0.7038274354$ $\mathrm{H}, 0,-1.096301366,2.2677409715,-1.6445679418$ C, $0,0.8688812964,0.4444884956,0.1743092818$ C, $0,-0.7558320042,2.3430747162,0.4887185446$ $\mathrm{H}, 0,-1.2177419865,1.9452376741,1.4013603577$ $\mathrm{H}, 0,-0.8318965604,3.436612412,0.5357684999$ C, $0,0.7157156878,1.9226688138,0.4428416296$ $\mathrm{H}, 0,1.2255377312,2.1716747966,1.3842834668$ $\mathrm{H}, 0,1.2376643878,2.4870813924,-0.3476137737$ C, $0,0.070156281,-1.8133429876,-0.5741909455$ $\mathrm{H}, \mathrm{O},-0.8580452445,-2.3445630704,-0.3294882383$ $\mathrm{H}, 0,0.267851638,-2.0303726029,-1.6334264432$ $\mathrm{N}, 0,2.0930313462,-0.0825036444,0.4898362979$ C, $0,2.4536822698,-1.4515148459,0.1404889585$ $\mathrm{H}, 0,3.2627805056,-1.7749100575,0.8050839086$ $\mathrm{H}, 0,2.8322342289,-1.5062414753,-0.8936683447$ C, $0,1.2218575775,-2.3471494745,0.2891353052$ $\mathrm{H}, 0,0.9250157234,-2.3611072586,1.3454083222$ $\mathrm{H}, 0,1.4797955432,-3.3747422414,0.0082264241$ $\mathrm{H}, 0,2.8477666397,0.5674982659,0.6650348425$ $\mathrm{H}, \mathrm{O},-4.1817375085,-0.2550230486,-0.0962404901$ $\mathrm{H}, \mathrm{O},-3.1920782633,-1.6844681371,0.418486957$ $\mathrm{H}, 0,-2.9595684462,-0.0858015269,1.2275979234$

Sum of electronic and zero-point Energies= Sum of electronic and thermal Energies= Sum of electronic and thermal Enthalpies= Sum of electronic and thermal Free Energies=
$-445.678589$

$-445.666906$ $-445.665961$ $-445.716460$

Insertion of 14 into isobutane, NImag=1, $-1065.9 \mathrm{~cm}^{-1}$ C, $0,0.7170981441,-0.7939262252,0.5518635561$ 
$C, 0,-0.4642368855,-0.6628117748,-0.2202358957$

$\mathrm{H}, 0,1.7074905608,-0.179871234,0.1070196362$

C, $0,2.3998776667,1.0770683344,-0.119387142$

C, $0,3.2884482374,1.2494371359,1.0973193382$

$\mathrm{H}, 0,3.7987944089,0.3129693115,1.3603779706$

$\mathrm{H}, 0,2.7255505629,1.5876684055,1.975331242$

$\mathrm{H}, 0,4.0767704476,2.0014868136,0.9132841078$

C, $0,3.1840862349,0.6403601509,-1.34614073$

$\mathrm{H}, 0,3.7070983098,-0.3090041247,-1.1710636519$

$\mathrm{H}, 0,3.9487791482,1.3889589321,-1.6182768886$

$\mathrm{H}, 0,2.5383111255,0.5066569516,-2.2221775042$

C, $0,1.47233189,2.2462998482,-0.3717866358$

$\mathrm{H}, 0,0.7498639698,2.032447267,-1.169263079$

$\mathrm{H}, 0,2.0337765549,3.1471651831,-0.6806872987$

$\mathrm{H}, 0,0.9035353696,2.515982056,0.5264492809$

C, $0,0.5772075091,-0.6674914298,2.0535052942$

$\mathrm{H}, 0,1.5057992152,-0.2857743898,2.489958808$

$\mathrm{H}, 0,0.4737059504,-1.6926384184,2.4501382173$

C, $0,-1.7168834615,-0.5787989331,0.3774732953$

C, $0,-0.6273047451,0.1653960719,2.5211961544$

$\mathrm{H}, 0,-0.4833077588,1.2129770853,2.2272744149$

$\mathrm{H}, 0,-0.7348845366,0.147196761,3.6131056454$

C, $0,-1.8966887931,-0.3751662671,1.8618533171$

$\mathrm{H}, \mathrm{O},-2.7498074123,0.2965611877,2.0335255395$

$\mathrm{H}, 0,-2.1680765345,-1.3400682269,2.3222805386$

C, $0,-0.3905092931,-0.829689024,-1.7337459055$

$\mathrm{H}, 0,0.4401639868,-0.2343174209,-2.1284909498$

$\mathrm{H}, 0,-0.147301633,-1.874130449,-1.9758472091$

$\mathrm{N}, 0,-2.879526314,-0.7267816016,-0.3296646247$

C, $0,-2.8901307327,-1.0887618309,-1.7413546908$

$\mathrm{H}, \mathrm{O},-3.838698014,-0.7539832595,-2.1763999532$

$\mathrm{H}, 0,-2.836142573,-2.1828136279,-1.8670189422$

C, $0,-1.696428853,-0.4281724147,-2.4332594426$

$\mathrm{H}, 0,-1.8268719558,0.6606989241,-2.3924778002$

$\mathrm{H}, 0,-1.6796152505,-0.7163648032,-3.4906857867$

$\mathrm{H}, 0,-3.7294512525,-0.8767935635,0.1974929579$

Sum of electronic and zero-point Energies= Sum of electronic and thermal Energies= Sum of electronic and thermal Enthalpies= Sum of electronic and thermal Free Energies=

$-563.538716$

$-563.522954$

$-563.522010$

$-563.581792$

Dimethoxycarbene (15), $\mathrm{C} 2 \mathrm{v}, \mathrm{NImag}=0$

$\mathrm{H}, 0,0.2 .17338483,1.30276924$

$\mathrm{C}, 0,0 ., 2.32013693,0.21986026$

$\mathrm{H}, 0,-0.89246991,2.87008195,-0.09174859$

$\mathrm{H}, 0,0.89246991,2.87008195,-0.09174859$

$0,0,0.1 .05154799,-0.44398886$

$\mathrm{C}, 0,0,0,0.37123105$

$0,0,0 .,-1.05154799,-0.44398886$

C, $0,0 .,-2.32013693,0.21986026$

$\mathrm{H}, \mathrm{O}, \mathrm{0} .,-2.17338483,1.30276924$

$\mathrm{H}, 0,0.89246991,-2.87008195,-0.09174859$

$\mathrm{H}, 0$, - $0.89246991,-2.87008195,-0.09174859$

Sum of electronic and zero-point Energies= Sum of electronic and thermal Energies= Sum of electronic and thermal Enthalpies= Sum of electronic and thermal Free Energies=

$-268.203327$

$-268.196959$

$-268.196014$

$-268.232136$ 
Insertion of 15 into acetonitrile, NImag=1, $-524.0 \mathrm{~cm}^{-1}$ C, $0,0.9105585707,-1.6861760215,-0.349460966$ $\mathrm{H}, 0,1.1327393448,-1.629773488,-1.4147541691$ C, $0,-0.7981977543,0.5147438302,-0.0318048034$ $\mathrm{H}, 0,-0.1509127376,-0.2898196221,0.3933795146$ C, $0,1.914517164,-1.1943789172,0.5049243097$ $\mathrm{N}, 0,2.6484625999,-0.6470866521,1.2438375923$ $\mathrm{H}, 0,0.4741860814,-2.6404884667,-0.0535399011$ $0,0,-0.320868177,1.5836135957,-0.5931188172$ $0,0,-2.0300032374,0.2641989018,-0.3885417283$ $\mathrm{C}, 0,1.0625867011,1.9332915818,-0.315340259$ $\mathrm{H}, 0,1.414983131,1.4560766928,0.6023385657$ $\mathrm{H}, \mathrm{0}, 1.082228284,3.0206691013,-0.2425934209$ $\mathrm{H}, 0,1.6650114368,1.5951157818,-1.1601808963$ C, $0,-2.6054168798,-0.9256440731,0.198094686$ $\mathrm{H}, \mathrm{O},-2.7716952855,-0.7712054049,1.2670378618$ $\mathrm{H}, 0,-1.9289264389,-1.7689829934,0.0311546971$ $\mathrm{H}, 0,-3.55416751,-1.0755034171,-0.3148988377$

Sum of electronic and zero-point Energies= Sum of electronic and thermal Energies= Sum of electronic and thermal Enthalpies= Sum of electronic and thermal Free Energies=

$-400.871535$

$-400.860936$

$-400.859992$

$-400.908430$

Insertion of 15 into methane, NImag=1, $-1203.6 \mathrm{~cm}^{-1}$ C, $0,-2.1443696354,-0.15350024,0.1564927824$ C, $0,0.0483342098,-0.0009171022,-0.6869572836$ $\mathrm{H}, \mathrm{0},-1.0127529345,0.1902561605,-1.0315468866$ $0,0,0.4760366496,-1.2555161351,-0.6267577117$ $0,0,0.6473906337,0.8328750731,0.1782056075$ C, $0,1.0816622531,-1.7273369176,0.5934755329$ $\mathrm{H}, 0,1.2864173515,-2.7850865581,0.4243065935$ $\mathrm{H}, 0,0.3876860434,-1.6050354282,1.4308928819$ $\mathrm{H}, 0,2.0110759064,-1.1917897932,0.8025653915$ C, $0,0.218091372,2.1903017088,0.0717673565$ $\mathrm{H}, 0,0.8520464519,2.7696475849,0.7442287388$ $\mathrm{H}, 0,-0.8312907681,2.2695199896,0.3794591297$ $\mathrm{H}, 0,0.3285431203,2.5514418967,-0.9562743543$ $\mathrm{H}, 0,-2.8914166358,0.6140499107,-0.0668398328$ $\mathrm{H}, \mathrm{O},-2.475517277,-1.1451293275,-0.1595249538$ $\mathrm{H}, \mathrm{0},-1.8645187216,-0.1380306332,1.2124797972$

Sum of electronic and zero-point Energies= Sum of electronic and thermal Energies= Sum of electronic and thermal Enthalpies= Sum of electronic and thermal Free Energies=

$-308.605725$

$-308.596663$ $-308.595719$ $-308.639398$

Insertion of 15 into methane, NImag=1, $-1181.6 \mathrm{~cm}^{-1}$ C, $0,-1.9881680319,-0.4699251479,-0.1177034711$ $\mathrm{C}, 0,0.3137378955,0.0703546899,-0.20290106$ $\mathrm{H}, 0,-0.5164534381,-0.1351604658,-0.9382177821$ $0,0,0.8860421431,-0.9099209198,0.4975212798$ $0,0,0.3738580258,1.2148111673,0.4800136365$ C, $0,1.0177861747,-2.1380285477,-0.2201710226$ $\mathrm{H}, 0,1.5869321414,-1.9897296317,-1.1443079898$ $\mathrm{H}, 0,1.5498365926,-2.8221924468,0.4419636773$ $\mathrm{H}, 0,0.0277971033,-2.5437268135,-0.4579277616$ C, $0,-0.1684089438,2.3365444954,-0.2174007895$ $\mathrm{H}, 0,-1.2479062504,2.2045528526,-0.354801221$ $\mathrm{H}, 0,0.3148051405,2.4602350413,-1.1929498539$ $\mathrm{H}, 0,0.0243831395,3.2083155637,0.4089018865$ 
$\mathrm{H}, 0,-2.1044595711,-1.5564931484,-0.1198272701$

$\mathrm{H}, 0,-2.0696879661,-0.0632312688,0.8921018967$

$\mathrm{H}, 0,-2.6941308105,0.0046353987,-0.8061568535$

Sum of electronic and zero-point Energies=

Sum of electronic and thermal Energies=

Sum of electronic and thermal Enthalpies=

Sum of electronic and thermal Free Energies=

$-308.605048$

$-308.595936$

$-308.594992$

$-308.638601$

Insertion of 15 into isobutane, NImag=1, $-1067.5 \mathrm{~cm}^{-1}$

C, $0,-1.4233129763,0.1073083905,-0.1736697229$

C, $0,0.9821407357,0.0809813479,-0.5807788789$

$\mathrm{H}, 0,0.0491343288,0.4816908129,-1.0372120604$

C, $0,-2.089695868,1.4088575016,-0.5855529456$

$\mathrm{H}, 0,-1.7204703295,2.2661677962,-0.0071498282$

$\mathrm{H}, 0,-3.1835733604,1.3714297565,-0.4229183816$

$\mathrm{H}, 0,-1.9412136303,1.6361063715,-1.6508268864$

C, $0,-1.8864558878,-1.0859084319,-0.9763564268$

$\mathrm{H}, 0,-2.9286781313,-1.3683836536,-0.7377543997$

$\mathrm{H}, 0,-1.2614549225,-1.9674175118,-0.7867004278$

$\mathrm{H}, 0,-1.8539168199,-0.8862198335,-2.0562348356$

$0,0,1.2353387351,-1.2244624897,-0.7286301646$

$0,0,1.4942320318,0.6314701192,0.5393656648$

C, $0,-1.4027401285,-0.105951283,1.3211581495$

$\mathrm{H}, 0,-1.0878533083,-1.123888513,1.5811834822$

$\mathrm{H}, 0,-2.4012915519,0.0440650322,1.7727536436$

$\mathrm{H}, \mathrm{O},-0.7188776959,0.5870118241,1.8331940035$

C, $0,1.7427395889,-1.9706259559,0.3916302962$

$\mathrm{H}, 0,1.8503903628,-2.9938831501,0.0293680726$

$\mathrm{H}, 0,1.0469398944,-1.9420492786,1.2348425269$

$\mathrm{H}, 0,2.7138348591,-1.581083998,0.7097736076$

C, $0,1.5557371115,2.0597276142,0.5165838786$

$\mathrm{H}, 0,2.0715508163,2.3588678697,1.4308739909$

$\mathrm{H}, 0,0.5490515422,2.4904936335,0.5063688396$

$\mathrm{H}, 0,2.1093863577,2.4046967053,-0.3635314488$

Sum of electronic and zero-point Energies= Sum of electronic and thermal Energies= Sum of electronic and thermal Enthalpies= Sum of electronic and thermal Free Energies=

$-426.464951$

$-426.451728$

$-426.450784$

$-426.504236$

Insertion of 15 into isobutane, NImag=1, $-1033.7 \mathrm{~cm}^{-1}$ C, $0,1.3555313589,-0.0534313748,0.0049825185$ C, $0,-1.0395264447,0.0368575583,-0.2770238318$ $\mathrm{H}, 0,-0.1301996772,-0.0206208111,-0.9280219697$ C, $0,2.1018025791,-0.8679498492,-1.0367868424$ $\mathrm{H}, 0,1.7768363543,-1.9158521072,-1.0561188891$ $\mathrm{H}, 0,3.1889079383,-0.8800353441,-0.8335459133$ $\mathrm{H}, 0,1.9800308558,-0.4604501274,-2.0502229882$ C, $0,1.7509774306,1.4043456611,0.0288292346$ $\mathrm{H}, 0,2.7689637234,1.5396063796,0.437342588$ $\mathrm{H}, 0,1.0767168239,1.9891650327,0.6674667818$ $\mathrm{H}, 0,1.7554098163,1.8502520553,-0.9754258529$ $0,0,-1.3153939314,1.1632347991,0.4093986143$ $0,0,-1.4472524393,-1.0364924512,0.4257910695$ C, $0,1.3124746359,-0.6822377204,1.3739981172$ $\mathrm{H}, 0,0.6768769998,-0.1073115094,2.0586704078$ $\mathrm{H}, 0,2.320149372,-0.7327092083,1.8252927937$ $\mathrm{H}, 0,0.9248938804,-1.7082770625,1.3496753503$ C, $0,-1.5446788413,2.3056800144,-0.4200209953$ $\mathrm{H}, 0$, - 2.4298483731,2.1523191193, - 1.0479599502 
$\mathrm{H}, \mathrm{0},-1.7082902352,3.1450986877,0.259050184$

$\mathrm{H}, 0,-0.6798408624,2.5180762398,-1.0577761886$

C, $0,-1.4468513836,-2.2544984557,-0.3163342475$

$\mathrm{H}, 0,-0.4255416854,-2.5635282693,-0.5667499128$

$\mathrm{H}, \mathrm{O},-2.0287124963,-2.1459387995,-1.2390935994$

$\mathrm{H}, \mathrm{O},-1.9035574783,-3.0063280602,0.3300359681$

Sum of electronic and zero-point Energies=

Sum of electronic and thermal Energies=

Sum of electronic and thermal Enthalpies=

$-426.464279$

$-426.451214$

$-426.450269$

Sum of electronic and thermal Free Energies=

$-426.503464$

Cyclopropenylidene (16), NImag=0

C, $0,0 ., 0 ., 1.2329938$

$\mathrm{C}, 0,0 .,-0.662543586,-0.033881416$

$\mathrm{C}, 0,0.0 .662543586,-0.033881416$

$\mathrm{H}, 0,0 .,-1.596176816,-0.582615484$

$\mathrm{H}, 0,0.1 .596176816,-0.582615484$

Sum of electronic and zero-point Energies=

115.319235

Sum of electronic and thermal Energies=

Sum of electronic and thermal Enthalpies=

$-115.316134$

$-115.315190$

Sum of electronic and thermal Free Energies=

$-115.342053$

Insertion of 16 into acetonitrile, NImag=1, $-520.6 \mathrm{~cm}^{-1}$ C, $0,-0.6350093468,0.9989769697,0.8333675777$

C, $0,-1.5635940701,0.8908182988,-0.1936742849$

C, $0,-1.3728267264,-0.1918225066,0.5748139291$

$\mathrm{H}, 0,0.4999842937,1.1914501211,0.9166493842$

C, $0,1.8187570375,0.3683699573,0.049082717$

$\mathrm{H}, 0,2.2100259222,1.0868925186,-0.6688102684$

$\mathrm{H}, 0,2.4735471761,0.1698205612,0.8962873136$

$\mathrm{H}, 0,-1.6756766763,-1.1712189086,0.9046343167$

$\mathrm{H}, 0,-2.0420327272,1.3360964646,-1.0548524203$

C, $0,1.1190767035,-0.7200983316,-0.4695121586$

$\mathrm{N}, 0,0.333675775,-1.5272152974,-0.822625001$

Sum of electronic and zero-point Energies=

Sum of electronic and thermal Energies=

Sum of electronic and thermal Enthalpies=

Sum of electronic and thermal Free Energies=

$-247.996986$

$-247.990528$

$-247.989584$

$-248.026696$

Insertion of 16 into methane, NImag=1, $-1275.6 \mathrm{~cm}^{-1}$

C, $0,0.1883255197,0.7637105275,0$.

C, $0,1.1314072121,-0.1087794449,0.6586043118$

C, $0,1.1314072121,-0.1087794449,-0.6586043118$

$\mathrm{H}, 0,-0.8746718278,1.0905338404,0$.

C, $0,-1.8017910091,-0.3054457007,0$.

$\mathrm{H}, 0,-1.6744080442,-0.900592598,-0.9066358811$

$\mathrm{H}, 0,-1.6744080442,-0.900592598,0.9066358811$

$\mathrm{H}, 0,-2.7568468143,0.2303030214,0$.

$\mathrm{H}, 0,1.5421205609,-0.4819436438,-1.5854990987$

$\mathrm{H}, 0,1.5421205609,-0.4819436438,1.5854990987$

Sum of electronic and zero-point Energies=

Sum of electronic and thermal Energies=

Sum of electronic and thermal Enthalpies=

155.722760

$-155.717223$

$-155.716279$

Sum of electronic and thermal Free Energies=

$-155.750491$ 
Insertion of 16 into isobutane, NImag=1, $-1130.9 \mathrm{~cm}^{-1}$ C, $0,-1.1287287085,-0.0297115621,1.1081186087$ C, $0,-2.1036223597,0.6837810586,0.3206608471$ C, $0,-2.1271201215,-0.6345743685,0.2613872965$ $\mathrm{H}, 0,-0.058864323,-0.0595626471,1.3480570783$ C, $0,0.8790044049,-0.0098352113,-0.1295698842$ $\mathrm{H}, 0,-2.6003015236,-1.5365342862,-0.097662297$ $\mathrm{H}, 0,-2.5438498464,1.6305660962,0.0447123333$ C, $0,2.116725938,-0.0715692931,0.7467335131$ $\mathrm{H}, 0,3.0462532029,-0.061189055,0.1488983298$ $\mathrm{H}, 0,2.1447772574,-0.9882805847,1.3522651519$ $\mathrm{H}, 0,2.1765189049,0.78523768,1.432390912$ C, $0,0.6626763171,-1.2302887574,-0.9854187314$ $\mathrm{H}, 0,1.3844278606,-1.2875797458,-1.8208502834$ $\mathrm{H}, 0,-0.33637032,-1.2250855747,-1.4510752914$ $\mathrm{H}, 0,0.7632982792,-2.1594725377,-0.4091545988$ C, $0,0.7078635269,1.2896534221,-0.8717514542$ $\mathrm{H}, 0,-0.2899143334,1.362046244,-1.3345132015$ $\mathrm{H}, 0,1.4326652556,1.3961091134,-1.6996907403$ $\mathrm{H}, 0,0.8405656022,2.1590135674,-0.2143385669$

Sum of electronic and zero-point Energies= Sum of electronic and thermal Energies= Sum of electronic and thermal Enthalpies= Sum of electronic and thermal Free Energies=

$-273.586930$

$-273.577522$

$-273.576577$

$-273.620544$

Carboxylate Carbene (17), NImag $=0$

C, $0,-1.1973687716,-0.2932485584,0.4612677482$

$\mathrm{H}, 0,-1.7997627255,-0.5746619504,-0.434228914$ C, $0,0.157791783,-0.1519325932,0.0129654074$ $0,0,1.2563648549,-0.6854136853,-0.1400266357$ $0,0,-0.2517117729,1.0911322928,-0.1613696168$

Sum of electronic and zero-point Energies= Sum of electronic and thermal Energies= Sum of electronic and thermal Enthalpies= Sum of electronic and thermal Free Energies=

$-227.154859$

$-227.151235$

$-227.150291$

$-227.180425$

Insertion of 17 into acetonitrile, frozen atoms C, - 1, - $1.5997287485,0.5167613609,-0.0532584204$ $\mathrm{H}, 0,-1.4760078361,0.7351918587,-1.1196253462$ C, $-1,0.8007195325,-0.5247244321,0.0581301546$ $\mathrm{H},-1,-0.4723770575,-0.0513852721,0.3391551736$ C, $0,-2.7695131099,-0.2763300416,0.1844692805$ $\mathrm{N}, 0,-3.6821194491,-0.9762159268,0.4050749864$ $\mathrm{H}, 0,-1.6387590584,1.4620992114,0.5004833174$ C, $0,1.9279962079,0.3167034283,-0.2359041541$ $\mathrm{H}, 0,0.7119037273,-1.3568048794,-0.6581199043$ $0,0,2.3748091783,1.2960909085,-0.807919887$ $0,0,2.4368449563,-0.564847324,0.6056649735$

Sum of electronic and zero-point Energies= Sum of electronic and thermal Energies= Sum of electronic and thermal Enthalpies= Sum of electronic and thermal Free Energies=

-359.881988
-359.874898
-359.873954
-359.915553

Insertion of 17 into methane, NImag=1, $-1045.5 \mathrm{~cm}^{-1}$ C, $0,0.2208235057,-0.8171503625,-0.596696173$ $\mathrm{H}, 0,1.3156845557,-0.34095326,-0.994818817$ 
$C, 0,-0.759736826,0.0685666313,0.0944303821$

$\mathrm{H}, 0,0.4596781115,-1.7120127663,-0.0084747782$

$0,0,-0.5464172828,1.2313100461,0.5074714536$

$0,0,-1.8384917279,-0.6075821773,0.0848976763$

C, $0,2.2059772574,0.1262249393,-0.1289169935$

$\mathrm{H}, 0,1.7075145231,0.8778034385,0.4814581857$

$\mathrm{H}, \mathrm{0}, 2.715063784,-0.6510261025,0.4437217666$

$\mathrm{H}, 0,2.8789474885,0.5705184917,-0.8737426904$

Sum of electronic and zero-point Energies= Sum of electronic and thermal Energies= Sum of electronic and thermal Enthalpies= Sum of electronic and thermal Free Energies=

$-267.606094$

$-267.600161$

$-267.599217$

$-267.635250$

Insertion of 17 into isobutane, NImag=1, $-973.1 \mathrm{~cm}^{-1}$ C, $0,-1.0634717148,-0.8121573379,-0.3373598604$ $\mathrm{H}, 0,0.1625437166,-0.8796152977,-0.4624489991$ C, $0,-1.7192900319,0.5130274177,-0.4588103205$ $\mathrm{H}, 0,-1.3044770505,-1.2979953005,0.6181730311$ $0,0,-1.1256714688,1.6213767979,-0.5323141977$ $0,0,-2.962134182,0.2720906999,-0.5469528616$ $\mathrm{C}, 0,0.9341401496,0.3546723259,1.5347389407$ $\mathrm{H}, 0,1.874630866,0.7580175803,1.9592281899$ $\mathrm{H}, 0,0.4632149284,-0.2624907333,2.3107303476$ $\mathrm{H}, 0,0.2700901268,1.1907501908,1.2910555036$ C, $0,1.8453861903,0.4268263216,-0.8067545473$ $\mathrm{H}, 0,2.79571111,0.8708560703,-0.4623348768$ $\mathrm{H}, 0,1.1372099825,1.2280050604,-1.0493388228$ $\mathrm{H}, 0,2.0568623873,-0.1505683872,-1.717473007$ C, $0,1.2159489826,-0.4401420194,0.2732028661$ C, $0,1.9326577156,-1.7579018459,0.5234223963$ $\mathrm{H}, 0,2.9468936448,-1.5919824861,0.9278228472$ $\mathrm{H}, 0,2.0389104128,-2.3417624125,-0.4008384008$ $\mathrm{H}, 0,1.3886273332,-2.3769034388,1.2489238116$

Sum of electronic and zero-point Energies= Sum of electronic and thermal Energies= Sum of electronic and thermal Enthalpies= Sum of electronic and thermal Free Energies=

$-385.471920$

$-385.462119$

$-385.461175$

$-385.506281$

Azacyclopentan-2-ylidene (18), NImag $=0$

$\mathrm{N}, 0,0.9206587605,-0.7446360087,-0.0698346458$ $C, 0,-0.241335589,-1.362043696,-0.0992825082$ C, $0,-1.2488354154,-0.2305897403,0.0703474679$ C, $0,-0.4936786643,1.1250490018,-0.0725849366$ C, $0,0.9803168708,0.7335336775,0.1170831203$ $\mathrm{H}, \mathrm{O},-2.0776391101,-0.3359249083,-0.6383624539$ $\mathrm{H}, 0,-1.6936263405,-0.352408803,1.0694753163$ $\mathrm{H}, 0,-0.8146523904,1.8794537113,0.6510914649$ $\mathrm{H}, 0,-0.6477653395,1.5424281982,-1.0732132256$ $\mathrm{H}, 0,1.3606029739,0.9628430046,1.1210430691$ $\mathrm{H}, 0,1.6591055674,1.186406767,-0.6137788916$ $\mathrm{H}, 0,1.7905601031,-1.2660413675,-0.1207916197$

Sum of electronic and zero-point Energies= Sum of electronic and thermal Energies= Sum of electronic and thermal Enthalpies= Sum of electronic and thermal Free Energies=

$-211.209314$ $-211.204675$ $-211.203730$ $-211.236787$ 
Insertion of 18 into acetonitrile, NImag=1, $-1053.7 \mathrm{~cm}^{-1}$ C, $0,0.3040800161,0.4188972143,-0.7292711747$ $\mathrm{H}, 0,-0.8827149917,0.9091738745,-0.7556658079$ C, $0,-2.3148367516,1.1929316135,-0.1999071871$ $\mathrm{H}, 0,-2.3421537254,1.9755519179,0.5609344085$ $\mathrm{N}, 0,0.4007545727,-0.7569086094,-0.1573554491$ $\mathrm{H}, 0,-0.438683084,-1.3292275328,-0.0196460294$ C, $0,1.6048899803,1.1507645412,-0.5041477138$ $\mathrm{H}, 0,1.9606490393,1.6339050368,-1.4201898612$ $\mathrm{H}, 0,1.4110347585,1.9598896432,0.2145072399$ C, $0,1.6636963628,-1.0398815292,0.5619800936$ $\mathrm{H}, 0,2.0172080228,-2.0468877821,0.323881722$ $\mathrm{H}, 0,1.4764979337,-0.9885691183,1.641343097$ C, $0,2.5855490776,0.0841622757,0.0524383258$ $\mathrm{H}, 0,3.227393556,-0.2912421288,-0.7505573113$ $\mathrm{H}, 0,3.2340647411,0.4713012822,0.8418736608$ C, $0,-2.5313187272,-0.1112271933,0.3031654962$ $\mathrm{N}, 0,-2.4878945314,-1.2305122578,0.6613162336$ $\mathrm{H}, 0,-2.9256762871,1.4441693448,-1.0697536508$

Sum of electronic and zero-point Energies= Sum of electronic and thermal Energies= Sum of electronic and thermal Enthalpies= Sum of electronic and thermal Free Energies=

$-343.903895$ $-343.895303$ $-343.894359$ $-343.938004$

Insertion of 18 into methane, NImag=1, $-1149.1 \mathrm{~cm}^{-1}$ C, $0,-0.5005271866,0.8723985553,-0.1887148014$ $\mathrm{H}, 0,-1.6303068765,0.8093585551,-0.3657309956$ C, $0,-2.3296931764,-0.6453424052,-0.1688957731$ $\mathrm{H}, 0,-2.7254614133,-0.7793267623,-1.1802641218$ $\mathrm{H}, 0,-1.7588503496,-1.5208481649,0.1455576789$ $\mathrm{H}, 0,-3.1207212441,-0.4065409923,0.5482554119$ $\mathrm{N}, 0,0.4057987964,0.4721605173,-1.1038206209$ $\mathrm{H}, 0,0.2739046472,0.6186986602,-2.0944505437$ $\mathrm{C}, 0,0.1065505954,0.5803304187,1.16199663$ $\mathrm{H}, 0,0.6358358006,1.4818986141,1.5060739871$ $\mathrm{H}, 0,-0.6403693632,0.3241114846,1.9152701733$ C, $0,1.511099353,-0.3543147912,-0.5964323336$ $\mathrm{H}, 0,2.4667267545,0.1770191714,-0.7059088974$ $\mathrm{H}, 0,1.5902078334,-1.3007691463,-1.1468161943$ C, $0,1.1209131853,-0.5598799322,0.8841943001$ $\mathrm{H}, 0,1.9911813375,-0.5389026409,1.5465606666$ $\mathrm{H}, 0,0.6272046746,-1.528973472,1.0053090491$

Sum of electronic and zero-point Energies= Sum of electronic and thermal Energies= Sum of electronic and thermal Enthalpies= Sum of electronic and thermal Free Energies=

$-251.628704$

$-251.621220$

$-251.620276$

$-251.660112$

Insertion of 18 into isobutane, NImag=1, $-960.7 \mathrm{~cm}^{-1}$ C, $0,0.4509552757,1.0938133435,0.2719321777$

$\mathrm{H}, \mathrm{O},-0.6739926378,1.0199193009,0.3382873339$ C, $0,-1.5770030856,-0.3696081712,0.0759822091$ $\mathrm{N}, 0,1.0771376648,1.0742978264,-0.9335578382$ $\mathrm{H}, 0,0.809937671,1.6980377853,-1.6822702711$ C, $0,1.3571344876,0.3779185692,1.2454641735$ $\mathrm{H}, 0,1.9081850207,1.1290435923,1.8332295539$ $\mathrm{H}, 0,0.8140383867,-0.2470584087,1.9576930749$ C, $0,2.3799346885,0.39811187,-0.9524826462$ $\mathrm{H}, 0,3.1988056463,1.1337044457,-0.945441669$ 
$\mathrm{H}, 0,2.4964111481,-0.2242114714,-1.847199009$ C, $0,2.3416001644,-0.4200789716,0.3510506681$ $\mathrm{H}, 0,3.3346642226,-0.5375746265,0.7942180464$ $\mathrm{H}, 0,1.939183555,-1.4174758452,0.1493383834$ C, $0,-2.6617182023,0.4948005327,-0.5391632842$ $\mathrm{H}, \mathrm{O},-2.3100710602,0.992442644,-1.4552436085$ $\mathrm{H}, 0,-3.0088421976,1.2770299131,0.1501942975$ $\mathrm{H}, 0,-3.5506315792,-0.0972539932,-0.8254711964$ C, $0,-1.9203246584,-0.8949053286,1.4498364945$ $\mathrm{H}, 0,-1.1097844329,-1.5041294926,1.8709707358$ $\mathrm{H}, 0,-2.821473394,-1.537773063,1.4421987963$ $\mathrm{H}, 0,-2.1293722137,-0.0797287607,2.1580114422$ C, $0,-1.0130580361,-1.3770813815,-0.8903807446$ $\mathrm{H}, 0,-0.3296571339,-2.0801333163,-0.3972774163$ $\mathrm{H}, 0,-0.4532447225,-0.8802026742,-1.6998079882$ $\mathrm{H}, \mathrm{O},-1.7992437357,-1.9825435881,-1.3799599265$

Sum of electronic and zero-point Energies= Sum of electronic and thermal Energies= Sum of electronic and thermal Enthalpies= Sum of electronic and thermal Free Energies=

$-369.489581$ $-369.478024$ $-369.477079$ $-369.526865$

N,N'-Dimethyl-1,3-diazacyclopent-4-en-2-ylidene (19), C2v, NImag=0 $\mathrm{C}, 0,0,0,0.98826803$

$\mathrm{N}, 0,0.1 .062643,0.11940201$

C, $0,0 ., 0.678277,-1.21816303$

$\mathrm{C}, 0,0 .,-0.678277,-1.21816303$

$\mathrm{N}, 0,0 .,-1.062643,0.11940201$

$\mathrm{H}, 0,0 ., 1.383412,-2.03685604$

$\mathrm{H}, 0,0 .,-1.383412,-2.03685604$

C, $0,0,-2.441959,0.5738$

$\mathrm{H}, 0,-0.89131785,-2.972806,0.21975741$

$\mathrm{H}, 0,0 .,-2.423948,1.66400089$

$\mathrm{H}, 0,0.89131785,-2.972806,0.21975741$

$\mathrm{C}, 0,0 ., 2.441959,0.5738$

$\mathrm{H}, 0,0.89131785,2.972806,0.21975741$

$\mathrm{H}, 0,0.2 .423948,1.66400089$

$\mathrm{H}, 0,-0.89131785,2.972806,0.21975741$

Sum of electronic and zero-point Energies=

Sum of electronic and thermal Energies=

Sum of electronic and thermal Enthalpies=

Sum of electronic and thermal Free Energies=

$$
\begin{aligned}
& -304.665454 \\
& -304.658455 \\
& -304.657510 \\
& -304.695698
\end{aligned}
$$

Insertion of 19 into acetonitrile, NImag=1, $-89.9 \mathrm{~cm}^{-1}$ $\mathrm{N}, 0,2.3796964334,0.3432246875,1.080001368$

$\mathrm{N}, 0,-1.4148329542,-0.5907424604,-0.1828235371$

C, $0,-1.9037743845,0.3315025144,0.7281495649$

$\mathrm{H}, 0,-2.7222261694,0.0969157902,1.3899067585$

C, $0,0.7895939042,2.1743462937,-0.8627889172$

$\mathrm{H}, 0,1.120591292,1.8805403124,-1.8605625729$

$\mathrm{H}, 0,0.355714287,3.1764432189,-0.9108676856$

C, $0,1.7172385202,-1.8919927842,-0.0269110301$

$\mathrm{H}, 0,0.2622923735,-0.5582559418,-1.5244141425$

$\mathrm{H}, 0,2.3164317026,-2.169305241,-0.8975159237$

$\mathrm{H}, 0,1.5771795293,-2.7344842828,0.6534187147$

C, $0,2.1294501317,-0.7064241306,0.6008133484$

C, $0,-0.386506324,-0.0297197969,-0.8468785632$

$\mathrm{N}, 0,-0.2424223822,1.2333471707,-0.4177456509$

C, $0,-1.1743772477,1.4691727076,0.5798048013$

$\mathrm{H}, 0$, - $1.2299103225,2.416257508,1.091906411$ 
$\mathrm{H}, 0,1.6274326665,2.1202937319,-0.1643414088$

C, $0,-1.6926188504,-2.0197235241,-0.2376849344$

$\mathrm{H}, 0,-0.7166471708,-2.52160631,-0.1300758703$

$\mathrm{H}, 0,-2.1564498409,-2.2881406926,-1.1906263664$

$\mathrm{H}, 0,-2.3655305242,-2.2824315578,0.5801212084$

Sum of electronic and zero-point Energies=

Sum of electronic and thermal Energies=

Sum of electronic and thermal Enthalpies=

Sum of electronic and thermal Free Energies=

$-437.340138$

$-437.329102$

$-437.328157$

$-437.377123$

Insertion of 19 into methane, NImag=1, $-916.2 \mathrm{~cm}^{-1}$ C, $0,-2.4960423493,0.4591651903,0.0590603745$ C, $0,-0.080832836,0.0434176641,-0.7247141954$ $\mathrm{H}, \mathrm{O},-1.0053388862,0.2355481764,-1.2699997348$ $\mathrm{N}, 0,0.29534744,-1.1631895692,-0.2042527518$ $\mathrm{N}, 0,0.6925709359,0.9935575579,-0.1185814637$ C, $0,-0.4608409828,-2.3724900486,-0.4520107832$ $\mathrm{H}, 0,-0.0283274003,-3.1947698908,0.1217276634$ $\mathrm{H}, 0,-0.432858288,-2.6295952897,-1.5153838213$ $\mathrm{H}, \mathrm{O},-1.5038233363,-2.2085892099,-0.1506594211$ C, $0,0.4183023031,2.4077756939,-0.2612697652$ $\mathrm{H}, 0,0.5510373277,2.7197473055,-1.3017981348$ $\mathrm{H}, 0,1.1060081004,2.97608561,0.3683911928$ $\mathrm{H}, 0,-0.6193071871,2.6015085989,0.0410152757$ C, $0,1.4193632981,0.3912048411,0.9087361782$ $\mathrm{H}, 0,2.0813638937,0.9597445208,1.5433294509$ C, $0,1.1743142791,-0.9395012614,0.8558655723$ $\mathrm{H}, 0,1.5818077872,-1.7532476556,1.4354824489$ $\mathrm{H}, \mathrm{O},-3.2144536426,-0.3072796082,-0.2616711629$ $\mathrm{H}, 0,-2.9002168935,1.4436510263,-0.2128442495$ $\mathrm{H}, 0,-2.3769023794,0.4071880183,1.1482457141$

Sum of electronic and zero-point Energies= Sum of electronic and thermal Energies= Sum of electronic and thermal Enthalpies= Sum of electronic and thermal Free Energies=

$-345.055175$ $-345.044535$ $-345.043591$ $-345.091855$

Insertion of 19 into isobutane, NImag=1, $-854.4 \mathrm{~cm}^{-1}$ C, $0,-1.8366377427,-0.2051600703,-0.0210665485$ $\mathrm{C}, 0,0.7287293859,0.0335577821,-0.6936483357$ $\mathrm{H}, 0,-0.1659960286,-0.0781085255,-1.2942157868$ C, $0,-2.2176724294,1.2592448511,-0.0683289024$ $\mathrm{H}, 0,-1.5231977263,1.8849172827,0.5145896877$ $\mathrm{H}, \mathrm{O},-3.2294562633,1.4602967543,0.3503496208$ $\mathrm{H}, 0,-2.2379000674,1.652850816,-1.0977871459$ C, $0,-2.7352532361,-1.0420930611,-0.9146586082$ $\mathrm{H}, 0,-3.8105064164,-0.9786294994,-0.6285787409$ $\mathrm{H}, 0,-2.4781892872,-2.1113376583,-0.8859856627$ $\mathrm{H}, 0,-2.6880460126,-0.7278157251,-1.9692675609$ $\mathrm{N}, 0,1.3764847988,-0.9949976898,-0.0637447518$ $\mathrm{N}, 0,1.2375779381,1.1971862867,-0.1781984112$ C, $0,-1.8073498084,-0.7128670479,1.4064770888$ $\mathrm{H}, 0,-1.5257078784,-1.7743255195,1.4730204956$ $\mathrm{H}, 0,-2.7955417214,-0.6289861649,1.914305041$ $\mathrm{H}, 0,-1.0964003951,-0.1499090397,2.0290549419$ C, $0,1.0217647586,-2.3818428461,-0.2699003708$ $\mathrm{H}, 0,1.4690659559,-2.9928945212,0.5167588795$ $\mathrm{H}, 0,1.3724134325,-2.7412267201,-1.2444172263$ $\mathrm{H}, \mathrm{O},-0.0702645358,-2.4668019301,-0.2273938665$ C, $0,1.0926356028,2.5032549966,-0.7953686579$ 
$\mathrm{H}, 0,1.8664119503,2.6795475019,-1.5557525759$

$\mathrm{H}, 0,1.1645301463,3.2778250944,-0.0272167858$

$\mathrm{H}, 0,0.1091210037,2.5715522837,-1.2616151661$

C, $0,2.1642683355,0.8791097077,0.8178291362$

$\mathrm{H}, 0,2.676219079,1.6433417332,1.3817826538$

C, $0,2.2328022183,-0.4710434434,0.8974147655$

$\mathrm{H}, 0,2.8052830991,-1.1085815526,1.5534739369$

Sum of electronic and zero-point Energies=

Sum of electronic and thermal Energies=

$-462.910609$

Sum of electronic and thermal Enthalpies=

$-462.896143$

Sum of electronic and thermal Free Energies=

$-462.895199$

$-462.952293$ 
(11) Gaussian 03, Frisch, M. J.; Trucks, G. W.; Schlegel, H. B.; Scuseria, G. E.; Robb, M. A.; Cheeseman, J. R.; Montgomery, Jr., J. A.; Vreven, T.; Kudin, K. N.; Burant, J. C.; Millam, J. M.; Iyengar, S. S.; Tomasi, J.; Barone, V.; Mennucci, B.; Cossi, M.; Scalmani, G.; Rega, N.; Petersson, G. A.; Nakatsuji, H.; Hada, M.; Ehara, M.; Toyota, K.; Fukuda, R.; Hasegawa, J.; Ishida, M.; Nakajima, T.; Honda, Y.; Kitao, O.; Nakai, H.; Klene, M.; Li, X.; Knox, J. E.; Hratchian, H. P.; Cross, J. B.; Adamo, C.; Jaramillo, J.; Gomperts, R.; Stratmann, R. E.; Yazyev, O.; Austin, A. J.; Cammi, R.; Pomelli, C.; Ochterski, J. W.; Ayala, P. Y.; Morokuma, K.; Voth, G. A.; Salvador, P.; Dannenberg, J. J.; Zakrzewski, V. G.; Dapprich, S.; Daniels, A. D.; Strain, M. C.; Farkas, O.; Malick, D. K.; Rabuck, A. D.; Raghavachari, K.; Foresman, J. B.; Ortiz, J. V.; Cui, Q.; Baboul, A. G.; Clifford, S.; Cioslowski, J.; Stefanov, B. B.; Liu, G.; Liashenko, A.; Piskorz, P.; Komaromi, I.; Martin, R. L.; Fox, D. J.; Keith, T.; Al-Laham, M. A.; Peng, C. Y.; Nanayakkara, A.; Challacombe, M.; Gill, P. M. W.; Johnson, B.; Chen, W.; Wong, M. W.; Gonzalez, C.; and Pople, J. A.; Gaussian, Inc., Pittsburgh PA, 2003. 